\title{
Antisense non-coding transcription represses the PHO5 model gene via remodelling of promoter chromatin structure
}

\author{
Ana Novačić ${ }^{1}$, Dario Menéndez ${ }^{2}$, Jurica Ljubas ${ }^{1}$, Slobodan Barbarić ${ }^{1}$, Françoise \\ Stutz ${ }^{2}$, Julien Soudet ${ }^{2^{*}}$, Igor Stuparević ${ }^{1^{*}}$ \\ ${ }^{1}$ Laboratory of Biochemistry, Department of Chemistry and Biochemistry, Faculty of \\ Food Technology and Biotechnology, University of Zagreb, Zagreb, Croatia \\ ${ }^{2}$ Department of Cell Biology, University of Geneva, 1211 Geneva 4, Switzerland
}

*Correspondence: istuparevic@pbf.hr (I.S.), julien.soudet@unige.ch (J.S.)

Running title: Antisense RNA represses $\mathrm{PHO5}$ gene

Keywords: PHO5, antisense transcription, chromatin, ncRNAs, RNA exosome

\begin{abstract}
Pervasive transcription of eukaryotic genomes generates non-coding transcripts with regulatory potential. We examined the effects of non-coding antisense transcription on the regulation of expression of the yeast PHO5 gene, a paradigmatic case for gene regulation through promoter chromatin remodeling. By enhancing or impairing the level of overlapping antisense transcription through specific mutant backgrounds and the use of the CRISPRi system, we demonstrated a negative role for antisense transcription at the PHO5 gene locus. Furthermore, enhanced elongation of $\mathrm{PHO5}$ antisense leads to a more repressive chromatin configuration at the PHO5 gene promoter, which is remodeled more slowly upon gene induction. The negative effect of antisense transcription on $\mathrm{PHO5}$ gene transcription is mitigated by inactivation of the histone deacetylase Rpd3, showing that PHO5 antisense RNA acts via histone deacetylation. This regulatory pathway leads to Rpd3-dependent decreased recruitment of the RSC chromatin remodeling complex to the PHO5 gene promoter upon induction of antisense transcription. Overall, we extend the model of $\mathrm{PHO}$ gene regulation by demonstrating a gene silencing function of antisense transcription through a chromatin-based mechanism.
\end{abstract}




\section{Introduction}

The canonical view of eukaryotic transcription has evolved from being considered a highly regulated process initiated from specialized genomic regions, such as gene promoters, to a process that permeates the entire genome (1). In addition to gene promoters, transcription often initiates from intergenic and intragenic regions, as well as regulatory regions such as gene enhancers. Most of the transcripts originating from these regions are non-coding RNAs usually rapidly degraded after their synthesis, suggesting that the act of transcription has more potential to exert important biological functions compared to the transcripts themselves (2).

In eukaryotic cells, promoter activation occurs in the context of a repressive chromatin structure, i.e. the packing of DNA with histone proteins into nucleosomal arrays (3). Since chromatinized DNA is not accessible for interaction with the transcriptional machinery, activators work in concert with chromatin-modifying and remodelling factors to expose regulatory sites and allow promoter activation. Chromatin modifiers catalyze covalent modifications of histones, such as acetylation, methylation, and phosphorylation, whereas chromatin remodelers use the energy of ATP hydrolysis to slide histones along the DNA or evict them from the DNA $(4,5)$. In gene-dense genomes such as that of yeast, transcription often initiates at the 3 ' end of genes, leading to the production of antisense (AS) non-coding transcripts (2). AS read-through transcription invades the promoter region of the corresponding gene, where it can exert regulatory effects that are usually repressive to transcription of the coding gene (6-9). Genome-wide and single gene studies have shown that promoters invaded by AS transcription read-through have high nucleosome occupancy and narrow nucleosome-depleted regions (NDRs) $(10,11)$. Our recent genome-wide study showed that induced elongation of non-coding antisense transcription into coding gene promoters results in increased deacetylation of promoter nucleosomes by Rpd3. Histone deacetylation leads to decreased recruitment of the major chromatin remodeler RSC and consequently to NDR closure, which represses transcription (12). However, there are still few examples of bona fide effects of specific AS RNAs on transcriptional regulation of their respective genes, such as the yeast $\mathrm{PHO} 84$ gene. 
Studies with the PHO84 gene have been highly instructive in elucidating the mechanisms of transcriptional regulation through AS non-coding RNAs (13-15). These studies converged on a model in which PHO84 AS transcription is rapidly terminated in wild-type cells by the NNS (Nrd1-Nab3-Sen1) complex and degraded by the activity of the Rrp6-containing nuclear RNA exosome. Inactivation of any of these crucial factors, such as in rrp6 $6 \Delta$ mutant cells, leads to transcriptional read-through of PHO84 AS transcripts, allowing recruitment of histone deacetylases (HDACs) Hda1 or Rpd3 to the PHO84 promoter. Histone deacetylation is thought to lock the chromatin structure of the promoter in a repressed configuration, thereby negatively regulating transcription of the sense transcript, i.e. PHO84 mRNA. This mechanism was subsequently explored genome-wide in yeast, which revealed a group of genes that in the absence of Rrp6 accumulate AS RNAs and are silenced in an HDAC-dependent manner (14). Genes of this class are characterized by AS transcripts that span the entire gene length, extend beyond the TSS and are enriched for so-called 'closed' promoters. These promoters are typical of inducible or stress-activated genes, and are characterized by precisely positioned nucleosomes whose remodeling is a prerequisite for transcriptional activation $(16,17)$. A paradigmatic closed promoter that also belongs to this gene class is that of the PHO5 gene, which is a member of the same $(\mathrm{PHO})$ regulon as $\mathrm{PHO} 84$ (18).

The PHO5 gene encodes the secreted non-specific acid phosphatase which is located in the periplasmic space and has a role in phosphate metabolism. Accordingly, expression of the PHO5 gene is regulated in response to intracellular phosphate concentration through the $\mathrm{PHO}$ signalling pathway, so that it is repressed when the intracellular concentration is abundant and induced under phosphate starvation conditions (18). This regulation is primarily achieved through phosphorylation of the specific activator Pho4. Under a high phosphate concentration Pho4 undergoes phosphorylation by the cyclin-dependent-kinase (Pho80-Pho85), accumulates in the nucleus, and activates transcription of the PHO5 gene. From the early days of chromatin research in the 1980s until now, the PHO5 gene promoter has been and continues to be instrumental in the discovery of numerous fundamental principles and mechanisms of chromatin structure remodeling (reviewed in (18)). In the repressed state, the $\mathrm{PHO} 5$ promoter features five precisely positioned nucleosomes, which upon 
induction are remodelled into a broad nucleosome-depleted region of $\sim 600 \mathrm{bp}$. This massive chromatin transition requires the concerted action of a large network of chromatin-modifying and -remodeling complexes as well as histone chaperones. The repressive chromatin configuration is maintained by $\mathrm{H} 3 \mathrm{~K} 4$ methylation catalyzed by Set1, a mark that recruits the histone deacetylase Rpd3 to the PHO5 promoter (19, 20). Another histone deacetylase, Hda1, plays a minor role in this process (21). When the intracellular phosphate concentration is limited, signal transduction via the $\mathrm{PHO}$ signaling pathway leads to the accumulation of the unphosphorylated transcriptional activator Pho4 in the nucleus $(18,22)$. The first step in transcriptional activation of the PHO5 gene is the binding of Pho4 to the UASp1 (Upstream activating sequence phosphate 1) site, which is located in the short nucleosome-depleted region between nucleosomes -2 and -3 of the PHO5 gene promoter. Pho4 recruits histone acetyltransferases, such as the Gcn5-containing SAGA complex, which establish a hyperacetylated promoter configuration $(23,24)$. Acetylated histones are read by chromatin-remodeling complexes containing bromodomains $(25,26)$. Alternatively, these remodelers can be recruited to the PHO5 promoter by direct interaction with Pho4 (27). Five remodelers (SWI/SNF, RSC, INO80, Isw1, Chd1) from all four yeast remodeler families cooperate to catalyze the chromatin opening at the $\mathrm{PHO} 5$ promoter $(28,29)$, with the most abundant remodeler, RSC, providing the crucial share of the remodeling activity required for this transition (29). Histone eviction allows Pho4 to bind to the UASp2 site otherwise covered by nucleosome -2, which is ultimatively required for full transcriptional activation (30-32).

Another layer of PHO5 promoter regulatory complexity was revealed upon mapping of the PHO5 AS transcript, CUT025 $(33,34)$. This transcript initiates from the 3 ' region of the PHO5 ORF and extends through its promoter region, spanning 2.4 $\mathrm{kb}$ in size. It is produced only in cells growing under repressive (phosphate-rich) conditions and is more abundant in rrp6 mutant compared to wild-type cells, indicating its degradation by the nuclear RNA exosome (33). AS transcription is generally associated with a repressive effect on transcription of the corresponding genes, and the PHO5 gene is among the rare examples for which AS transcription is proposed to have a positive effect (33). In this work, we examined the effect of noncoding AS transcription on PHO5 gene expression by enhancing or impairing 
elongation of the PHO5 AS transcript. In both cases, our results argue in favour of antisense transcription having a negative effect on PHO5 gene transcription. Moreover, we provide evidence that this negative effect occurs through a chromatinbased mechanism mediated by AS transcription which decreases the accessibility of the chromatin structure at the PHO5 gene promoter.

\section{Materials and methods}

Yeast strains and primer sequences used in this study are listed in Supplementary Tables S1 and S2, respectively.

\section{Strains, media, plasmids and strain construction}

Yeast Saccharomyces cerevisiae strains used in this study are listed in Supplementary Table S1. All strains were grown at $30^{\circ} \mathrm{C}$. For repressive conditions (high phosphate, $+\mathrm{P}_{\mathrm{i}}$ ), yeast strains were grown in YNB medium supplemented with $1 \mathrm{~g} / \mathrm{l} \mathrm{KH}_{2} \mathrm{PO}_{4}$ (YNBP) with or without lack of amino acids for plasmid selection. For gene induction by phosphate starvation $\left(-\mathrm{P}_{\mathrm{i}}\right)$, cells were washed in water and resuspended in the phosphate-free synthetic medium with or without lack of amino acids for plasmid selection $(28,29,35)$. Anchor-away of Nrd1-AA and Sth1-AA was induced by adding $1 \mu \mathrm{g} / \mathrm{ml}$ of rapamycin (Sigma) to the medium. The RRP6 gene was deleted using a disruption cassette generated by PCR with primer pairs RRP6-Kan1 and RRP6-Kan2 or RRP6hph_fwd and RRP6hph_rev. Transformants were selected on G-418 (0.2 $\mathrm{mg} / \mathrm{ml}$, Sigma) or Hygromycin B $(0.3 \mathrm{mg} / \mathrm{ml}$, Sigma) plates, depending on the marker, and gene deletion was confirmed by PCR. The pP5Z reporter plasmid is centromeric vector that carries a PHO5 promoter-lacZ gene fusion and is described in (36). The pCEN-RRP6 plasmid was previously constructed by Gateway cloning from the pAG416GPD backbone (37). Plasmid pTDH3-dCas9 (pFS3891) (38) was obtained from Addgene (Plasmid \#46920). Plasmid pFS3892, which contains the guide RNA scaffold, was generated by one-step isothermal Gibson assembly reaction (New England BioLabs) using two fragments, one obtained by PCR on pRPR1_gRNA_handle_RPR1t (Addgene Plasmid \#49014) using OFS_2869 and OFS_2870 oligonucleotides, the other by PCR on YCpLac33 using OFS_2871 and OFS_2872 oligonucleotides. Plasmid PHO5 AS gDNA-URA3 was then obtained by 
Gibson assembly reaction (NEB) using OFS_2886 and OFS_2887 to amplify pFS3892 backbone and OFS_2888 and OFS_3095 for gDNA cloning.

\section{Enzyme activity assays, RNA isolation, Nothern blot and RT-qPCR}

Acid phosphatase and beta-galactosidase activity assays were done with intact yeast cells, exactly as described in (29). Total RNA was extracted by the hot phenol method (39), treated with RNAse-free DNAse I (New England Biolabs) and purified by phenol/chloroform extraction. Strand-specific reverse transcription was performed using $1 \mu \mathrm{g}$ of RNA and strand-specific oligonucleotides $(0.1 \mu \mathrm{M}$ each) with the ProtoScript First Strand cDNA Synthesis Kit (New England Biolabs) supplemented with actinomycin $D$ (Sigma) to final concentration $5 \mu \mathrm{g} / \mathrm{ml}$ to ensure strand specificity. cDNAs were amplified in Roche LightCycler 480 with the Maxima SYBR Green qPCR Master Mix detection kit (Thermo Scientific). The qPCR datasets were analysed using the $\triangle \triangle \mathrm{Ct}$ method, and the results were normalized to either PMA1, ACT1 or SCR1 RNAs amplification, which were used as internal controls. Amplifications were done in duplicate for each sample, and three independent RNA extractions were analysed. For the Northern blot, total RNA (10 $\mu$ g for each sample) was run on a $1 \%$ denaturing formaldehyde agarose gel and transferred to nylon membranes (Amersham Hybondtm-N+). Membranes were crosslinked and incubated overnight at $60^{\circ} \mathrm{C}$ with $100 \mu \mathrm{g} / \mathrm{ml}$ boiled salmon sperm DNA in 50\% formamide, $5 \mathrm{x}$ standard saline citrate (SSC), $20 \%$ dextran sulfate sodium, $1 \%$ sodium dodecyl sulfate (SDS). Subsequently, membrane wered hybridized with 32P-labeled SP6/T7 riboprobes in 50\% formamide, $7 \%$ SDS, $0.2 \mathrm{M} \mathrm{NaCl}, 80 \mathrm{mM}$ sodium phosphate $(\mathrm{pH} 7.4)$, and $100 \mu \mathrm{g} / \mathrm{ml}$ boiled salmon sperm DNA for $6 \mathrm{~h}$. All blots were washed with 2 X SSC and $0.1 \%$ SDS for 5 minutes at $60^{\circ} \mathrm{C}$ and then with $0.5 \mathrm{XSC}$ and $0.1 \%$ SDS for 45 minutes at $60^{\circ} \mathrm{C}$. Riboprobes were obtained by SP6/T7 in vitro transcription of gene-specific PCR fragments containing an SP6/T7 promoter. Quantifications were performed with a Phosphor Imager machine.

\section{Chromatin analysis}

For anti-histone $\mathrm{H} 3$ chromatin immunoprecipitation (ChIP), forty millilitres of cells were fixed with $1 \%$ formaldehyde for $20 \mathrm{~min}$. After glycine addition to stop the reaction, the cells were washed and lysed with glass beads to isolate chromatin. The cross-linked 
chromatin was sheared by sonication with a Vibra-Cell sonicator to reduce average fragment size to approximately 500 base pairs. Chromatin fractions of $400 \mu \mathrm{l}$ were taken for each immunoprecipitation reaction and incubated with $4 \mu \mathrm{l}$ of anti-histone $\mathrm{H} 3$ antibodies (ab1791, Abcam) at $4^{\circ} \mathrm{C}$ overnight. After incubation, $40 \mu \mathrm{l}$ of protein $\mathrm{G}$ PLUS-agarose beads (sc-2002, Santa Cruz Biotechnology) were added and incubated at $4^{\circ} \mathrm{C}$ for $2 \mathrm{~h}$. The beads were washed extensively, and the chromatin was eluted. Eluted supernatants (output) and the input controls were hydrolysed with Pronase $\left(0.8 \mathrm{mg} / \mathrm{ml}\right.$ final concentration, Sigma) at $42^{\circ} \mathrm{C}$ for $2 \mathrm{~h}$, followed by incubation at $65^{\circ} \mathrm{C}$ for $7 \mathrm{~h}$ to reverse cross-linked DNA complexes. DNA was extracted using the Macherey Nagel Nucleospin Gel \& PCR Cleanup Kit. The immunoprecipitated DNAs (output) were quantified by qPCR in Roche LightCycler 480 with the Maxima SYBR Green qPCR Master Mix detection kit (Thermo Scientific). Amplifications were done in triplicate for each sample. Immunoprecipitated samples (output) were normalized to input and to a PHO5-adjacent control region which does not show chromatin signatures similar to the PHO5 gene promoter, as described in (31). Chromatin analysis of yeast nuclei by restriction nuclease accessibility assay was done as described previously $(29,35,40) .120 \mathrm{U}$ of the Clal restriction enzyme (New England Biolabs) was used for chromatin analysis of nuclei and $40 \mathrm{U}$ of Haelll (New England Biolabs) was used for secondary cleavage. Probe for hybridization was as described previously $(29,35,41)$. Quantification of the percentage of cleaved DNA was done by Phosphorlmager analysis (Fuji FLA3000). ChIP of dCas9 was essentially perfomed as in (12) without addition a $S$. pombe spike-in. An anti-Cas9 antibody (Diagenode \#C15310258) was used for the immunoprecipitation step.

\section{Downloaded data sets}

For RNA-seq and RNAPII PAR-CLIP, data were retrieved from (42) (GEO: GSE175991) and from (43) (GEO: GSE56435). Data of MNase-seq, ATAC-seq and Sth1 ChEC-seq were reanalyzed from (12) (GEO: GSE130946). 


\section{Results}

\section{AS transcription is involved in regulation of $\mathrm{PHO5}$ gene expression}

The product of antisense transcription at the PHO5 model gene locus, CUT025 (hereafter referred to as PHO5 AS), is initiated at the 3 ' end of the gene ORF in the antisense direction and extends through the PHO5 promoter region (Fig. 1A). The 3'5 ' exoribonuclease Rrp6, which is the catalytic subunit of the nuclear RNA exosome complex, degrades this transcript in wild-type (wt) cells, consistent with the increased level of this transcript in rrp6 4 mutant cells (Fig. S1A). We confirmed the increased level of the PHO5 AS transcript at the PHO5 promoter region in rrp6 4 compared to wt cells by strand-specific reverse-transcription quantitative PCR (RT-qPCR) upon shifting the cells from repressive (phosphate-rich, + $\mathrm{P}_{\mathrm{i}}$; YNB with additional $1 \mathrm{~g} / \mathrm{l}$ $\mathrm{KH}_{2} \mathrm{PO}_{4}$ ) to inducing (no phosphate, $-\mathrm{P}_{\mathrm{i}}$ ) conditions. PHO5 AS accumulation in rrp64 was most pronounced under repressive conditions (Fig. 1A, $0 \mathrm{~h}$ of induction), consistent with (33). After shifting to inducing conditions, the level of PHO5 AS gradually decreased in both wild-type and rrp6 $\Delta$ cells, however the increased level in rrp6 $\Delta$ cells was still present at an early time point of gene induction (Fig. 1A). The PHO5 AS transcript has a much lower steady-state level than the corresponding $\mathrm{PHO5}$ mRNA transcript, as observed by RNA-seq, which measures steady-state RNA levels, i.e., takes into account both the level of nascent transcription and RNA degradation. However, the RNAPII photoactivatable ribonucleoside-enhanced crosslinking and immunoprecipitation (PAR-CLIP) signal, which measures only nascent transcription, is comparable or even higher for the AS transcript than for the mRNA transcript under same growth conditions $(4 \mathrm{mM} \mathrm{Pi})$, showing that the AS transcript is being produced to a potentially significant level (Fig. 1B). Whole-genome tiling array datasets revealed production of another non-coding transcript at the PHO5 gene locus, SUT446, transcribed in the sense direction through the PHO5 promoter region, which appears not to be accumulated in rrp6 mutant cells and is weakly expressed ((14, 44); Fig. S1A). It was determined by RT-qPCR that the level of SUT446 was not significantly increased in rrp6 $6 \Delta$ compared to wild-type cells neither in repressive nor inducing conditions (Fig. S1B), arguing against its gene-regulatory function. Overall, these data 
support a possible regulatory role of the CUT025 AS non-coding transcript, but not the SUT446 promoter non-coding transcript, in regulation of PHO5 gene transcription.

We further investigated whether the increased level of the PHO5 AS transcript under repressive conditions and during early gene induction in rrp6 $\Delta$ cells correlates with a change in PHO5 mRNA level. PHO5 mRNA was quantified by RT-qPCR upon gene induction and a strong delay in its expression was observed in rrp6 4 cells compared to wild-type cells (Fig. 1C). This delay persisted during the first hours of gene induction and corresponded to a delay in expression of the Pho5 acid phosphatase, as determined by measuring its enzymatic activity (Fig. 1D). However, after prolonged induction, the level of acid phosphatase in rrp6 $\Delta$ cells reached that of wild-type cells (Fig. 1D). The observed delay in gene expression was dependent on the catalytic activity of Rrp6, because the catalytically dead rrp6Y361A mutant cells also exhibited delayed PHO5 gene expression, and acid phosphatase activity was brought to wild-type levels when a functional RRP6 gene was expressed from a centromeric plasmid in rrp6 4 cells (Fig. S2A). A similar delay was also measured with rrp6 $\Delta$ cells of two other genetic backgrounds (Figs. S2B and S2C), showing that it is not specific to the W303-derived strain used in these experiments. We also performed a control experiment to test whether the observed kinetic delay in $\mathrm{PHO} 5$ expression in rrp6 $\Delta$ cells is caused by an indirect effect due to compromised signal transduction through the $\mathrm{PHO}$ signaling pathway. We made use of a construct in which expression of the lacZ reporter gene was driven by the PHO5 promoter and monitored its expression by measuring beta-galactosidase activity upon induction (no phosphate, $\mathrm{P}_{\mathrm{i}}$ ) in wild-type and rrp6 4 cells (Fig. S2D). Expression kinetics of the PHO5 promoterlacZ construct were similar in wild-type and rrp6 4 cells, arguing that $\mathrm{PHO}$ signaling is not compromised in rrp6 4 cells. This result demonstrates that the kinetic delay in PHO5 expression observed with the rrp6 $\Delta$ strain (Figs.1C and D) was not an indirect effect caused by compromised induction strength and consequently impaired PHO5 transcriptional activation. Additionally, this result speaks in favour of a possible regulatory role of the AS transcript originating from the PHO5 ORF.

Rrp6 is the nuclear-specific catalytic subunit of the RNA exosome complex. To determine the involvement of other RNA exosome subunits and cofactors in the 
regulation of PHO5 gene expression, we examined the kinetics of PHO5 gene expression using appropriate mutant cells. Deletion mutants for the monomeric cofactors of the nuclear exosome, Rrp47 and Mpp6, also showed delayed acid phosphatase expression kinetics (Fig. 2A). The TRAMP complex is another cofactor of the nuclear RNA exosome and consists of a non-canonical poly(A) polymerase (Trf4 or Trf5), an RNA-binding subunit (Air1 or Air2), and the essential helicase Mtr4 (45, 46). Interestingly, single air1 $\Delta$ and air2 $\Delta$ mutant cells showed no delay, whereas the air1 $\Delta$ air2 $\Delta$ double mutant showed an even greater delay than the rrp6 $\Delta$ mutant (Fig. $2 \mathrm{~A}$ ), consistent with a high degree of redundancy between homologous TRAMP subunits (47). The mutant for the exonuclease activity of the essential RNA exosome catalytic subunit Dis3 (dis3 $4+$ pDis3-exo-) also showed delayed kinetics compared with the corresponding wild type cells (dis $3 \Delta+p D i s 3)$ and with the mutant for its endoribonuclease activity (dis3 $\Delta+p$ Dis3-endo-) (Fig. 2B). These results demonstrate the involvement of the second catalytic subunit of the RNA exosome, Dis3, as well as the nuclear RNA exosome cofactors Rrp47, Mpp6 and the TRAMP complex in the regulation of $\mathrm{PHO5}$ gene expression.

In rrp6 6 and other RNA exosome deletion mutant backgrounds, AS transcription is constitutively induced due to sequestration of the NNS (Nrd1-Nab3Sen1) termination complex by stabilised non-coding RNAs. The NNS complex cannot be efficiently recycled to sites of transcription, inducing termination defects at noncoding RNA loci and resulting in their increased elongation frequency (48). To rule out possible indirect effects on transcription of the PHO5 gene due to gene deletion mutant backgrounds in which AS transcription is constitutively elongated, we turned to a system in which AS elongation is inducible. To this end, we used the Anchor Away (AA) system to rapidly deplete Nrd1 protein from the nucleus by rapamycin treatment (49). Since Nrd1 belongs to the NNS surveillance system, its removal is expected to trigger transcriptional read-through of non-coding RNAs (48). Indeed, treatment of Nrd1-AA cells with rapamycin resulted in rapid induction of the PHO5 AS transcript production, clearly demonstrating that the NNS complex is important for its early termination in wild-type cells. Importantly, even under PHO5 repressive conditions, induction of PHO5 AS transcript production through the Nrd1-AA system was accompanied by downregulation of PHO5 mRNA levels, as shown by Northern blot 
(Fig. 3A). Furthermore, with the Nrd1-AA system, it was possible to induce elongation of AS transcription by adding rapamycin simultaneously when shifting the cells to PHO5 inducing conditions (i.e. phosphate free medium ) (Fig. 3B) or an hour before the shift (Fig. 3C). Consistently, the kinetics of Pho5 expression monitored by measuring acid phosphatase activity showed a kinetic delay which was dependent on the timing of rapamycin addition during cultivation (Figs. $3 \mathrm{~B}$ and $3 \mathrm{C}$ ). The results of this experiment demonstrated that the negative correlation between PHO5 AS and mRNA transcript levels is not an indirect consequence of gene deletion mutant backgrounds, since it is also seen upon induced Nrd1 depletion.

\section{Block of AS transcription through dCas9 enhances the kinetics of PHO5 gene expression}

Given that accumulation of the PHO5 AS transcript negatively affects $\mathrm{PHO5}$ gene transcription kinetics, blocking AS transcript production should enhance it. To specifically target $\mathrm{PHO5}$ AS transcription, we undertook a CRISPRi approach in which a catalytically dead Cas9 protein (dCas9) is directed by a guide RNA (gRNA) to interfere with AS transcription at the PHO5 gene locus. The CRISPRi system blocks transcription due to physical collision between the elongating RNA Polymerase and the dCas9:gRNA complex(50). Furthermore, this system was shown to function in a strand-specific manner, by blocking transcription only when the nontemplate DNA strand of a transcription unit is targeted $(50,51)$. Therefore, we targeted dCas 9 to the nontemplate strand of the AS transcription unit at the PHO5 gene locus to block only AS transcription. First, we confirmed the presence of the dCas9 protein at the PHO5 ORF by anti-Cas9 chromatin immunoprecipitation (ChIP). Notably, a strong peak of dCas9 binding at the PHO5 gene ORF compared to a control strain not expressing the gRNA was observed (Fig. 4A), while no dCas9 binding could be detected at the PHO5 promoter region covered by nucleosomes -4 and -1 (Fig. 4A). RNA levels in the Nrd1AA strain with the active CRISPRi system were monitored by RT-qPCR and showed a highly reproducible decrease in PHO5 AS levels compared to the control strain (Fig. 4B). This decrease was significant at the PHO5 promoter and ORF regions without rapamycin addition or with rapamycin (i.e., depletion of Nrd1 which induces AS transcription). These results are consistent with a dCas9-mediated transcriptional 
roadblock of AS transcription at the PHO5 gene locus. After the addition of rapamycin, PHO5 AS levels were increased in both the CRISPRi Nrd1-AA strain and the control Nrd1-AA strain. However, its levels in the CRISPRi strain remained significantly lower, maintaining the difference in levels already observed without the addition of rapamycin (Fig. 4B). These results demonstrated that the dCas9-mediated roadblock of AS transcription at the PHO5 gene locus is robust and maintained after global induction of AS transcription, although AS transcription was not completely abolished.

Importantly, impairment of PHO5 AS RNA elongation led to an increase in PHO5 mRNA levels (Fig. 4C), clearly demonstrating the direct role of AS transcription in PHO5 gene repression. Also, it argues in favour that the CRISPRi system strandspecifically blocked only AS transcription without significantly impacting mRNA transcription. We further tested if impairment of AS transcription with use of the CRISPRi system, would result in enhanced kinetics of PHO5 gene expression. As expected, the kinetics of PHO5 gene expression upon gene induction, were slightly faster when AS transcript production was impaired by dCas9 than in the control strain (Figs. 4D and 4E). This effect was noticed only at very early timepoints of gene induction (30 min for mRNA levels and 1,5 h for acid phospatase levels), possibly due to the dCas9 protein losing its roadblock function past a certain level of ongoing transcription.

\section{AS RNA elongation affects PHO5 promoter chromatin structure}

Since transcriptional activation of the PHO5 promoter requires a large transition of its chromatin structure, we investigated whether the kinetics of PHO5 promoter chromatin opening upon gene induction also inversely correlate with PHO5 AS transcription. To this end, we examined the chromatin structure at the PHO5 promoter with anti-histone H3 ChIP at nucleosome -2, which covers the high-affinity Pho4 binding site and is considered the critical nucleosome for PHO5 chromatin remodeling (18). A higher histone occupancy was observed in rrp6 $\Delta$ and air1 $\Delta$ air2 $\Delta$ compared to wild-type cells already under repressive conditions (Fig. 5A). Accordingly, histone removal from the PHO5 promoter was slower in rrp64 than in wild-type cells during the first hours of gene induction and reached a similar final level after 5 hours (Fig. 
$5 A)$. To confirm the delayed kinetics of chromatin opening in rrp6 $\Delta$ cells, we took advantage of the Clal restriction enzyme accessibility assay, which quantifies the efficiency of cleavage by Clal enzyme at nucleosome -2 of the PHO5 promoter (Fig. 5B). Consistent with the anti-histone H3 ChIP, the accessibility of the Clal site at the PHO5 promoter was lower in rrp6 4 and air1 $\Delta a i r 2 \Delta$ than in wild type cells during the first hours of gene induction (Fig. 5C). These results show that AS transcription mediates a negative effect on PHO5 transcriptional activation by influencing the chromatin structure at its promoter region.

Our results suggest that AS transcription at the PHO5 gene locus locks the chromatin structure of the $\mathrm{PHO} 5$ promoter in a more repressive configuration that is harder to remodel (Fig. 5). This could be due to the activity of HDACs, which have been shown to negatively affect chromatin structure at the PHO5 promoter $(20,21)$. Remarkably, inactivation of the HDAC Rpd3 in the rrp6 $\triangle$ mutant background does not affect the level of the PHO5 AS RNA, but it restores transcription activation of the PHO5 gene to the level or even higher than in wild-type cells, as shown by RNAsequencing of single and double deletion mutant cells ((14); Fig. 6A). Accordingly, the expression kinetics of acid phosphatase measured with the rpd3 $\Delta$ rrp6 $\Delta$ double mutant cells are not delayed compared to wild-type cells, in contrast to the corresponding rrp6 $\Delta$ single mutant cells (Fig. 6B). These results suggest that the PHO5 AS transcript acts via a pathway that involves histone deacetylation. Gcn5, the catalytic subunit of the SAGA and ADA complexes, is known to be the major histone acetyltransferase that enables physiological gene induction kinetics at the PHO5 promoter $(23,24)$. We reasoned that in the absence of Gcn5, i.e. when the majority of histone acetylation normally present at the PHO5 gene promoter is reduced, an rrp6 $\Delta$ strain should have no additional effect on the kinetics of PHO5 gene expression. Indeed, the kinetics of acid phosphatase expression in gcn54rrp6 $\Delta$ double mutant strain are the same as in the gcn5 single mutant (Fig. 6C). Taken together, these results argue that AS transcription represses the PHO5 gene via histone deacetylation.

Histone acetylation plays two important roles in transcriptional activation. It neutralizes the positive change of lysine groups, thereby weakening histone-DNA interactions, but also provides docking sites for the bromodomains of proteins involved 
in transcriptional regulation. The major chromatin remodeling complex required for PHO5 promoter chromatin opening, RSC (Remodels Structure of Chromatin) (29), contains seven out of fourteen bromodomains identified in $S$. cerevisiae $(26,52)$. Therefore, we wondered whether AS-induced deacetylation of the PHO5 promoter may inhibit the recruitment of RSC, resulting in a more closed chromatin conformation. To this end, we examined the PHO5 gene locus in our recent Micrococcal Nuclease sequencing (MNase-seq), Assay for Transposase-Accessible Chromatin using sequencing (ATAC-seq) and Sth1-Chromatin Endogenous Cleavage-sequencing (Sth1-ChEC-seq) datasets performed with Nrd1-AA and Nrd1-AA rpd3 $\Delta$ cells with and without the addition of rapamycin (12). The ChEC-seq data show the fold change in association of Sth1, the ATP-ase subunit of RSC, with chromatin upon induction of AS transcription (-Rap vs +Rap) (Fig. 7A). In addition, the ATAC-seq data provide us with information about chromatin accessibility under the same conditions. In Nrd1-AA cells there is a negative fold change, i.e. a decrease in Sth1 binding, associated with a decrease in chromatin accessibility upon addition of rapamycin, for the region encompassing nucleosome -2 of the PHO5 promoter (Fig. 7A). Conversely, in isogenic rpd3 $\Delta$ cells, addition of rapamycin has almost no effect on Sth1 binding or chromatin accessibility in this region (Fig. 7A). These data argue in favour of a model in which read-through of AS transcription acts via recruitment of histone deacetylases to the PHO5 gene promoter, the activity of which results in decreased recruitment of the RSC complex. RSC is the most abundant and the only essential chromatin remodeling complex in yeast $(53,54)$. Therefore, we used the anchor away system to deplete its catalytic subunit Sth1 from the nucleus. Rapamycin was added to phosphate-free medium, which causes the depletion of Sth1 simultaneously with the induction of the PHO5 gene. This limits the amount of RSC available for recruitment at the early stages of gene induction, which should further aggravate the decrease in local chromatin acetylation caused by $\mathrm{PHO5}$ AS readthrough (Fig. 7B). Consistently, anchoring away of Sth1-AA combined with rrp6 deletion showed a synthetic effect on the kinetics of PHO5 gene induction (Fig. 7C). 


\section{Discussion}

The role of non-coding RNAs in regulation of gene expression could not be appreciated until recent advances in high-throughput methods facilitated their detection and characterization. From a gene-centered view, non-coding RNAs can be transcribed in tandem with genes, i.e. from the same strand as the gene, or from the opposite strand, resulting in production of antisense (AS) non-coding RNAs. Apart from a few isolated examples, production of AS non-coding RNAs is generally thought to have a repressive cis-regulatory effect on the expression of associated mRNAs (6, $8,55)$. This seems to be particularly the case when transcription of AS non-coding RNAs invades promoters of coding genes $(9,14,56)$. In light of this current view, we felt compelled to reexamine the role of AS transcription at the model yeast $\mathrm{PHO}$ gene locus, which was originally suggested to support gene activation (33). In this work, we show a clear negative role for AS transcription in PHO5 gene expression. By leveraging mutant backgrounds in which AS transcription is constitutively enhanced or induceable, we show that increased PHO5 AS elongation frequency correlates with decreased expression of the corresponding mRNA. Furthermore, we demonstrate that the use of a CRISPRi system that specifically blocks AS transcription at the PHO5 gene locus increases the level of PHO5 mRNA and enhances its induction kinetics upon phosphate depletion. Importantly, these observations show that AS RNA transcription has an impact on $\mathrm{PHO} 5$ gene expression in wild-type cells, and not only upon enhanced AS RNA stabilisation in strains mutant for RNA degradation factors. The role of PHO5 AS transcription is therefore reminiscent of the role of AS transcription in maintaining the tight repression of quiescence-related transcripts during the exponential growth phase, recently demonstrated by Nevers et al. (9). A previous study suggesting a positive regulatory role for PHO5 AS transcription achieved AS inactivation by incorporating a full-length marker gene sequence with its promoter region in the middle of the PHO5 gene ORF (33). This major perturbation of the PHO5 gene locus may have resulted in experimental artefacts, highlighting the need for precise interventions, such as those achieved by the CRISPRi system, to perform functional analyses of AS transcripts (57).

There are now several well-described examples of yeast gene loci at which either antisense or upstream non-coding transcription that extends through a coding 
sense promoter has an inhibitory effect on its transcription initiation $(13,58-61)$. In most cases, it is likely that elongation of non-coding transcription leads to directly to displacement of transcription factors (TFs) and/or the preinitiation complex (PIC) or that the recruitment of TFs or the PIC to these gene promoters is decreased as a consequence of a more repressive chromatin configuration established at the promoter region due to elongation of non-coding transcription (see (62) for a review). This model is supported by whole-genome analyses showing that invasion of gene promoters by AS transcription leads to increased histone occupancy and altered recruitment of chromatin-modifying and -remodeling complexes $(10,12,63)$. At the tandemly transcribed SRG1 IncRNA/SER3 protein-coding gene locus, non-coding transcription has been shown to cause nucleosome deposition at the gene promoter, thereby repressing SER3 transcription (64). As another example, we have shown that AS transcription at the PHO84 gene locus silences the corresponding gene by recruiting HDACs to its promoter region (13). The AS RNA does not recruit the HDACs directly, but the act of its transcription promotes a histone methylation-based mechanism to restore the repressive chromatin structure in the wake of the elongating RNA Pol II. The histone methyltransferase Set2 associates with the elongating RNA Pol II and catalyses H3K36 methylation, a mark read by the Eaf3 chromodomain of the HDAC Rpd3 (65). Consistent with this, our recent genome-wide study in yeast has shown that AS transcription leads to deacetylation of a subpopulation of $-1 /+1$ nucleosomes associated with increased H3K36 methylation, which in turn leads to decreased binding of the RSC chromatin-remodeling complex and sliding of nucleosomes into previously nucleosome-depleted regions (12). We have now shown that elongation of PHO5 AS under repressive conditions leads to increased histone occupancy at the PHO5 gene promoter and slower histone removal upon gene induction. Moreover, the negative effect of AS RNA elongation on PHO5 gene activation is mitigated by inactivation of Rpd3, suggesting a histone acetylation-based regulatory mechanism that may affect the recruitment of RSC, a chromatin remodeler that plays an important role in PHO5 gene promoter opening (29). This is supported by ChEC-seq of Sth1, the catalytic subunit of RSC, showing a decrease in its recruitment to the $\mathrm{PHO5}$ gene promoter upon induction of antisense transcription, that is suppressed by inactivation of Rpd3. 
PHO5 belongs to a group of $\sim 100$ genes that are more transcribed in AS direction as a non-coding transcript than in the sense orientation as a mRNA in a standard medium (Fig. 1B). In such culture conditions, the Pho4 transcriptional activator is rarely located in the nucleus (18). Thus, as we proposed in (42) for the SAGA-dependent gene class to which PHO5 belongs, the steady-state chromatin structure of the promoter NDR might be maintained tightly closed by ongoing AS transcription.

The regulatory roles of non-coding RNAs are intertwined with that of chromatin structure. Not only does non-coding transcription affect chromatin structure, but chromatin structure also determines where and how often non-coding RNAs are transcribed. This fact is increasingly appreciated with respect to the directionality of transcription at promoters of coding genes. Specifically, chromatin modifiers such as the HDAC Hda1, and chromatin remodelers such as RSC, have been shown to dictate promoter directionality by attenuating divergent non-coding transcription $(66,67)$. Furthermore, chromatinization of DNA limits aberrant transcription that would otherwise occur on naked DNA, as was recently demonstrated through in vitro experiments by the Kornberg group (68). In this study, a chromatinized PHO5 gene locus fragment was transcribed seven times more from the physiological transcription start site than the same naked DNA locus, and also resulted in transcription patterns more similar to those seen in vivo. Although only chromatin was considered in this study, it would be interesting to also investigate non-coding transcription using a similar in vitro transcription system.

Chromatin remodeling complexes and non-coding RNAs are important regulators of gene expression, and therefore dysregulation of either of these factors may affect the development and progression of various cancers. The SWI/SNF family of chromatin remodeling complexes includes the SWI/SNF complex with its catalytic subunits BRG1 or BRM in humans, and the SWI/SNF and RSC complex with their catalytic subunits Snf2 and Sth1, respectively, in yeast. Numerous associations between chromatin remodelers of this family and long non-coding RNAs have been detected in human cancers (reviewed in (69)). These complexes and the corresponding regulatory non-coding RNAs therefore represent promising diagnostic and therapeutic targets. Transcription of long non-coding RNAs is particularly 
important for the yeast genome, which has a very high gene density, such that many of them overlap coding gene ORFs or promoter regions. Another reason why budding yeast is a good model for studying the transcription of such long ( $\geq 200 \mathrm{nt}$ ) non-coding RNAs is that it exclusively synthesizes this non-coding transcript class since its divergence from other yeasts and the loss of the RNAi system that produces short non-coding RNAs (70). In addition, extensively studied gene loci, such as the yeast PHO5 gene, are invaluable for mechanistic studies of gene regulation. Studies of the PHO5 gene and its promoter region made an immense contribution to deciphering the mechanisms of gene regulation through chromatin remodeling (18) and our study now opens the possibility to focus on non-coding transcription in this system.

\section{Funding:}

This work was supported by funds from the Swiss National Science Foundation (grant 31003A_182344) to F.S. and by Croatian Science Foundation Grant UIP-2017-054411 to I. S.

\section{Acknowledgements:}

I.S. and A.N. dedicate this paper to A. Rachid Rahmouni, who always provided support and encouragement. We are grateful to $P$. Korber for extensively commenting on the manuscript and providing help with the restriction nuclease accessibility assay.

Declaration of competing interest: The authors declare no competing interests.

\section{References}

1. Jensen,T.H., Jacquier,A. and Libri,D. (2013) Dealing with pervasive transcription. Mol. Cell, 52, 473-484.

2. Murray,S.C. and Mellor,J. (2016) Using both strands: The fundamental nature of antisense transcription. Bioarchitecture, 6, 12-21.

3. Baldi,S., Korber,P. and Becker,P.B. (2020) Beads on a string-nucleosome array arrangements and folding of the chromatin fiber. Nat. Struct. Mol. Biol., 27, 109_ 118.

4. Desjarlais,R. and Tummino,P.J. (2016) Role of Histone-Modifying Enzymes and Their Complexes in Regulation of Chromatin Biology. Biochemistry, 55, 15841599.

5. Clapier,C.R. and Cairns,B.R. (2009) The biology of chromatin remodeling complexes. Annu. Rev. Biochem., 78, 273-304.

6. Pelechano,V. and Steinmetz,L.M. (2013) Gene regulation by antisense 
bioRxiv preprint doi: https://doi.org/10.1101/2022.02.21.481265; this version posted February 21, 2022. The copyright holder for this

transcription. Nat. Rev. Genet., 14, 880-893.

7. Donaldson,M.E. and Saville,B.J. (2012) Natural antisense transcripts in fungi. Mol. Microbiol., 85, 405-417.

8. Wery,M., Gautier,C., Descrimes,M., Yoda,M., Vennin-Rendos,H., Migeot,V., Gautheret,D., Hermand,D. and Morillon,A. (2018) Native elongating transcript sequencing reveals global anti-correlation between sense and antisense nascent transcription in fission yeast. Rna, 24, 196-208.

9. Nevers,A., Doyen,A., Malabat,C., Néron,B., Kergrohen,T., Jacquier,A. and Badis,G. (2018) Antisense transcriptional interference mediates conditionspecific gene repression in budding yeast. Nucleic Acids Res., 46, 6009-6025.

10. Murray,S.C., Haenni,S., Howe,F.S., Fischl,H., Chocian,K., Nair,A. and Mellor,J. (2015) Sense and antisense transcription are associated with distinct chromatin architectures across genes. Nucleic Acids Res., 43, 7823-7837.

11. Dai,Z. and Dai,X. (2012) Antisense transcription is coupled to nucleosome occupancy in sense promoters. Bioinformatics, 28, 2719-2723.

12. Gill,J.K., Maffioletti,A., García-Molinero,V., Stutz,F. and Soudet,J. (2020) Fine Chromatin-Driven Mechanism of Transcription Interference by Antisense Noncoding Transcription. Cell Rep., 31.

13. Camblong,J., Iglesias,N., Fickentscher,C., Dieppois,G. and Stutz,F. (2007) Antisense RNA Stabilization Induces Transcriptional Gene Silencing via Histone Deacetylation in S. cerevisiae. Cell, 131, 706-717.

14. Castelnuovo,M., Zaugg,J.B., Guffanti,E., Maffioletti,A., Camblong,J., Xu,Z., Clauder-Münster,S., Steinmetz,L.M., Luscombe,N.M. and Stutz,F. (2014) Role of histone modifications and early termination in pervasive transcription and antisense-mediated gene silencing in yeast. Nucleic Acids Res., 42, 4348-4362.

15. Castelnuovo,M., Rahman,S., Guffanti,E., Infantino,V., Stutz,F. and Zenklusen,D. (2013) Bimodal expression of PHO84 is modulated by early termination of antisense transcription. Nat. Struct. Mol. Biol., 20, 851-858.

16. Zaugg,J.B. and Luscombe,N.M. (2012) A genomic model of condition-specific nucleosome behavior explains transcriptional activity in yeast. Genome Res., 22, 84-94.

17. Cairns,B.R. (2009) The logic of chromatin architecture and remodelling at promoters. Nature, 461, 193-8.

18. Korber,P. and Barbaric,S. (2014) The yeast PHO5 promoter: from single locus to systems biology of a paradigm for gene regulation through chromatin. Nucleic Acids Res., 42, 10888-10902.

19. Carvin,C.D. and Kladde,M.P. (2004) Effectors of lysine 4 methylation of histone $\mathrm{H} 3$ in Saccharomyces cerevisiae are negative regulators of PHO5 and GAL110. J. Biol. Chem., 279, 33057-33062.

20. Wang,S.-S., Zhou,B.O. and Zhou,J.-Q. (2011) Histone H3 Lysine 4 Hypermethylation Prevents Aberrant Nucleosome Remodeling at the PHO5 Promoter. Mol. Cell. Biol., 31, 3171-3181.

21. Vogelauer,M., Wu,J., Suka,N. and Grunstein,M. (2000) Global histone acetylation and deacetylation in yeast. Nature, 4, 276-84.

22. Austin,S. and Mayer,A. (2020) Phosphate Homeostasis - A Vital Metabolic Equilibrium Maintained Through the INPHORS Signaling Pathway. Front. Microbiol., 11, 1-21.

23. Barbaric,S., Walker,J., Schmid,A., Svejstrup,J.Q. and Hörz,W. (2001) Increasing the rate of chromatin remodeling and gene activation - A novel role for the histone acetyltransferase Gcn5. EMBO J., 20, 4944-4951. 
bioRxiv preprint doi: https://doi.org/10.1101/2022.02.21.481265; this version posted February 21, 2022. The copyright holder for this

preprint (which was not certified by peer review) is the author/funder, who has granted bioRxiv a license to display the preprint in perpetuity. It is made available under aCC-BY-NC-ND 4.0 International license.

24. Barbaric,S., Reinke,H. and Hörz,W. (2003) Multiple mechanistically distinct functions of SAGA at the PHO5 promoter. Mol. Cell. Biol., 23, 3468-3476.

25. Hassan,A.H., Prochasson,P., Neely,K.E., Galasinski,S.C., Chandy,M., Carrozza,M.J. and Workman,J.L. (2002) Function and Selectivity of Bromodomains in Anchoring Chromatin-Modifying Complexes to Promoter Nucleosomes. Cell, 111, 369-379.

26. Chen,G., Li,W., Yan,F., Wang,D. and Chen,Y. (2020) The Structural Basis for Specific Recognition of H3K14 Acetylation by Sth1 in the RSC Chromatin Remodeling Complex. Structure, 28, 111-118.e3.

27. Neely,K.E., Hassan,A.H., Brown,C.E., Howe,L. and Workman,J.L. (2002) Transcription Activator Interactions with Multiple SWI/SNF Subunits. Mol. Cell. Biol., 22, 1615-1625.

28. Barbaric,S., Luckenbach,T., Schmid,A., Blaschke,D., Hörz,W. and Korber,P. (2007) Redundancy of chromatin remodeling pathways for the induction of the yeast PHO5 promoter in vivo. J. Biol. Chem., 282, 27610-27621.

29. Musladin,S., Krietenstein,N., Korber,P. and Barbaric,S. (2014) The RSC chromatin remodeling complex has a crucial role in the complete remodeler set for yeast PHO5 promoter opening. Nucleic Acids Res., 42, 4270-4282.

30. Venter,U., Svaren,J., Schmitz,J., Schmid,A. and Hörz,W. (1994) A nucleosome precludes binding of the transcription factor Pho4 in vivo to a critical target site in the PHO5 promoter. EMBO J., 13, 4848-4855.

31. Reinke,H. and Hörz,W. (2003) Histones are first hyperacetylated and then lose contact with the activated PHO5 promoter. Mol. Cell, 11, 1599-1607.

32. Boeger,H., Griesenbeck,J., Strattan,J.S. and Kornberg,R.D. (2003) Nucleosomes unfold completely at a transcriptionally active promoter. Mol. Cell, 11, 1587-98.

33. Uhler,J.P., Hertel,C. and Svejstrup,J.Q. (2007) A role for noncoding transcription in activation of the yeast PHO5 gene. Proc. Natl. Acad. Sci. U. S. A., 104, 80118016.

34. Xu,Z., Wei,W., Gagneur,J., Perocchi,F., Clauder-Münster,S., Camblong,J., Guffanti,E., Stutz,F., Huber,W. and Steinmetz,L.M. (2009) Bidirectional promoters generate pervasive transcription in yeast. Nature, 457, 1033-7.

35. Almer,A., Rudolph,H., Hinnen,A. and Hörz,W. (1986) Removal of positioned nucleosomes from the yeast $\mathrm{PHO} 5$ promoter upon $\mathrm{PHO} 5$ induction releases additional upstream activating DNA elements. EMBO J., 5, 2689-2696.

36. Straka,C. and Horz,W. (1991) A functional role for nucleosomes in the repression of a yeast promoter. EMBO J., 10, 361-368.

37. Stuparevic,I., Mosrin-Huaman,C., Hervouet-Coste,N., Remenaric,M. and Rahmouni,A.R. (2013) Cotranscriptional recruitment of RNA exosome cofactors Rrp47p and Mpp6p and two distinct Trf-Air-Mtr4 polyadenylation (TRAMP) complexes assists the exonuclease Rrp6p in the targeting and degradation of an aberrant messenger ribonucleoprotein particle (mRN. J. Biol. Chem., 288, 31816-31829.

38. Gilbert,L.A., Larson,M.H., Morsut,L., Liu,Z., Brar,G.A., Torres,S.E., SternGinossar,N., Brandman,O., Whitehead,E.H., Doudna,J.A., et al. (2013) CRISPR-mediated modular RNA-guided regulation of transcription in eukaryotes. Cell, 154, 442.

39. Schmitt,M.E., Brown,T.A. and Trumpower,B.L. (1990) A rapid and simple method for preparation of RNA from Saccharomyces cerevisiae. Nucleic Acids Res., 18, 3091-3092.

40. Gregory,P.D., Barbaric,S. and Hörz,W. (1999) Restriction nucleases as probes 
bioRxiv preprint doi: https://doi.org/10.1101/2022.02.21.481265; this version posted February 21,2022 . The copyright holder for this

for chromatin structure. Methods Mol. Biol., 119, 417-25.

41. Almer,A. and Hörz,W. (1986) Nuclease hypersensitive regions with adjacent positioned nucleosomes mark the gene boundaries of the $\mathrm{PHO} 5 / \mathrm{PHO} 3$ locus in yeast. EMBO J., 5, 2681-2687.

42. Soudet,J., Beyrouthy,N., Pastucha,A.M. and Maffioletti,A., Bakir, Z., Stutz, F. (2021) Antisense-mediated repression of SAGA-dependent genes involves the HIR histone chaperone. BioRxiv doi: 10.1101/2021.07.05.451174

43. Schaughency,P., Merran,J. and Corden,J.L. (2014) Genome-Wide Mapping of Yeast RNA Polymerase II Termination. PLoS Genet., 10.

44. Xu,Z., Wei,W., Gagneur,J., Perocchi,F., Clauder-Münster,S., Camblong,J., Guffanti,E., Stutz,F., Huber,W. and Steinmetz,L.M. (2009) Bidirectional promoters generate pervasive transcription in yeast. Nature, 457, 1033-1037.

45. LaCava,J., Houseley,J., Saveanu,C., Petfalski,E., Thompson,E., Jacquier,A. and Tollervey,D. (2005) RNA degradation by the exosome is promoted by a nuclear polyadenylation complex. Cell, 121, 713-724.

46. Schmidt,K. and Butler,J.S. (2013) Nuclear RNA surveillance: Role of TRAMP in controlling exosome specificity. Wiley Interdiscip. Rev. RNA, 4, 217-231.

47. Delan-Forino,C., Spanos,C., Rappsilber,J. and Tollervey,D. (2020) Substrate specificity of the TRAMP nuclear surveillance complexes. Nat. Commun., 11.

48. Villa,T., Barucco,M., Martin-Niclos,M.J., Jacquier,A. and Libri,D. (2020) Degradation of Non-coding RNAs Promotes Recycling of Termination Factors at Sites of Transcription. Cell Rep., 32.

49. Haruki,H., Nishikawa,J. and Laemmli,U.K. (2008) The Anchor-Away Technique: Rapid, Conditional Establishment of Yeast Mutant Phenotypes. Mol. Cell, 31, 925-932.

50. Qi,L.S., Larson,M.H., Gilbert,L.A., Doudna,J.A., Weissman,J.S., Arkin,A.P. and Lim,W.A. (2013) Repurposing CRISPR as an RNA-yuided platform for sequence-specific control of gene expression. Cell, 152, 1173-1183.

51. Lenstra,T.L., Coulon,A., Chow,C.C. and Larson,D.R. (2015) Single-Molecule Imaging Reveals a Switch between Spurious and Functional ncRNA Transcription. Mol. Cell, 60, 597-610.

52. Zhang,Q., Chakravarty,S., Ghersi,D., Zeng,L., Plotnikov,A.N., Sanchez,R. and Zhou,M.-M. (2010) Biochemical profiling of histone binding selectivity of the yeast bromodomain family. PLoS One, 5, e8903.

53. Ghaemmaghami,S., Huh,W.-K., Bower,K., Howson,R.W., Belle,A., Dephoure,N., O'Shea,E.K. and Weissman,J.S. (2003) Global analysis of protein expression in yeast. Nature, 425, 737-41.

54. Cairns,B.R., Lorch,Y., Li,Y., Zhang,M., Lacomis,L., Erdjument-Bromage,H., Tempst,P., Du,J., Laurent,B. and Kornberg,R.D. (1996) RSC, an essential, abundant chromatin-remodeling complex. Cell, 87, 1249-1260.

55. Statello,L., Guo,C.J., Chen,L.L. and Huarte,M. (2021) Gene regulation by long non-coding RNAs and its biological functions. Nat. Rev. Mol. Cell Biol., 22, 96118.

56. Huber,F., Bunina,D., Gupta,I., Khmelinskii,A., Meurer,M., Theer,P., Steinmetz,L.M. and Knop,M. (2016) Protein Abundance Control by Non-coding Antisense Transcription. Cell Rep., 15, 2625-2636.

57. Liu,S.J. and Lim,D.A. (2018) Modulating the expression of long non-coding RNA $\mathrm{s}$ for functional studies. EMBO Rep., 19, 1-11.

58. Van Werven,F.J., Neuert,G., Hendrick,N., Lardenois,A., Buratowski,S., Van Oudenaarden,A., Primig,M. and Amon,A. (2012) Transcription of two long 
bioRxiv preprint doi: https://doi.org/10.1101/2022.02.21.481265; this version posted February 21,2022 . The copyright holder for this

noncoding RNAs mediates mating-type control of gametogenesis in budding yeast. Cell, 150, 1170-1181.

59. Hongay,C.F., Grisafi,P.L., Galitski,T. and Fink,G.R. (2006) Antisense Transcription Controls Cell Fate in Saccharomyces cerevisiae. Cell, 127, 735745.

60. Schulz,D., Schwalb,B., Kiesel,A., Baejen,C., Torkler,P., Gagneur,J., Soeding,J. and Cramer,P. (2013) Transcriptome surveillance by selective termination of noncoding RNA synthesis. Cell, 155, 1075.

61. Du Mee,D.J.M., Ivanov,M., Parker,J.P., Buratowski,S. and Marquardt,S. (2018) Efficient termination of nuclear Incrna transcription promotes mitochondrial genome maintenance. Elife, 7, 1-24.

62. Soudet,J. and Stutz,F. (2019) Regulation of Gene Expression and Replication Initiation by Non-Coding Transcription: A Model Based on Reshaping Nucleosome-Depleted Regions: Influence of Pervasive Transcription on Chromatin Structure. BioEssays, 41.

63. Brown,T., Howe,F.S., Murray,S.C., Wouters,M., Lorenz,P., Seward,E., Rata,S., Angel,A. and Mellor,J. (2018) Antisense transcription-dependent chromatin signature modulates sense transcript dynamics. Mol. Syst. Biol., 14, 1-21.

64. Hainer,S.J., Pruneski,J.A., Mitchell,R.D., Monteverde,R.M. and Martens,J.A. (2011) Intergenic transcription causes repression by directing nucleosome assembly. Genes Dev., 25, 29-40.

65. Lee,J.S. and Shilatifard,A. (2007) A site to remember: H3K36 methylation a mark for histone deacetylation. Mutat. Res. - Fundam. Mol. Mech. Mutagen., 618, 130-134.

66. Gowthaman,U., Ivanov,M., Schwarz,I. and Patel,H.P. (2021) The Hda1 histone deacetylase limits divergent non-coding transcription and restricts transcription initiation frequency. EMBO J., 10.15252/embj.2021108903.

67. Wu,A., Vivori,C., Patel,H., Sideri,T. and van Werven,F.J. (2021) RSC-mediated nucleosome positioning and GRFs form barriers in promoters to limit divergent noncoding transcription. bioRxiv.

68. Nagai,S., Davis,R.E., Mattei,P.J., Eagen,K.P. and Kornberg,R.D. (2017) Chromatin potentiates transcription. Proc. Natl. Acad. Sci., 114, 1536-1541.

69. Tang,Y., Wang,J., Lian,Y., Fan,C., Zhang,P., Wu,Y., Li,X., Xiong,F., Li,X., Li,G., et al. (2017) Linking long non-coding RNAs and SWI/SNF complexes to chromatin remodeling in cancer. Mol. Cancer, 16, 1-8.

70. Alcid,E.A. and Tsukiyama,T. (2016) Expansion of antisense IncRNA transcriptomes in budding yeast species since the loss of RNAi. Nat. Struct. Mol. Biol., 23, 450-455.

71. Lardenois,A., Liu,Y., Walther,T., Chalmel,F., Evrard,B., Granovskaia,M., Chu,A., Davis,R.W., Steinmetz,L.M. and Primig,M. (2011) Execution of the meiotic noncoding RNA expression program and the onset of gametogenesis in yeast require the conserved exosome subunit Rrp6. Proc. Natl. Acad. Sci. U. S. A., 108, 1058-1063.

Figure descriptions:

Figure 1. Kinetics of PHO5 gene induction are inversely correlated with level of the corresponding antisense transcript. (A) Scheme showing transcription of an antisense (AS) RNA at the PHO5 gene locus (left) and its levels at the PHO5 promoter 
region in wild type BMA41 (wt) and corresponding rrp6 4 mutant cells upon induction through phosphate starvation, monitored by strand-specific reverse transcription quantitative PCR (RT-qPCR) (right). RT-qPCR values were normalized to PMA1 mRNA and expressed relative to transcript abundance in wild-type cells under repressive conditions ( $0 \mathrm{~h}$ of induction), which was set to 1. (B) The left panel shows RNA-seq signal from an Nrd1-AA strain in the absence of rapamycin (wild-type equivalent) at the PHO5 locus. The right panel represents RNAPII PAR-CLIP signal or nascent transcription signal in the same conditions. Data were retrieved from (42) and (43), respectively. (C) Levels of PHO5 mRNA in wild-type BMA41 (wt) and corresponding rrp6 4 mutant cells upon induction through phosphate starvation. RTqPCR values were normalized to PMA1 mRNA and expressed relative to transcript abundance in wild-type cells at repressive conditions ( $0 \mathrm{~h}$ of induction), which was set to 1. (D) Same as (C), but acid phosphatase induction kinetics were monitored by measuring acid phosphatase activity with whole cells. Reported values represent the means and standard deviations of three independent experiments $(n=3)$.

\section{Figure 2. Induction of the PHO5 gene is negatively affected in RNA exosome}

mutant cells. (A) Acid phosphatase induction kinetics in wild-type BMA41 (wt) and corresponding deletion mutant cells for Rrp6 and RNA exosome cofactors upon induction through phosphate starvation. Reported values represent the means and standard deviations of three independent experiments $(n=3)$. (B) Same as $(A)$, but for W303-derived strains with genomic copy of DIS3 gene deleted but bearing a centromeric plasmid that carries the wild type copy of DIS3 gene (dis $3 \Delta+p D i s 3)$ or its alleles with abolished endonuclease (dis3 $4+$ pDis3-endo-, D171N) or exonuclease (dis3 $4+$ pDis3-exo', D551N) activity.

\section{Figure 3. Induction of PHO5 AS elongation by depletion of Nrd1 from the nucleus} delays induction of the PHO5 gene. (A) Nothern blot analysis of total RNA from the parental Anchor Away (AA) and the corresponding Nrd1-AA strains upon addition of rapamycin to the growth medium. Nothern blots were probed specifically for sense and antisense PHO5 transcripts, while ACT1 mRNA was used as a loading control. (B) Acid phosphatase induction kinetics in Nrd1-AA strain upon induction through phosphate starvation with (+Rap) or without addition of rapamycin (-Rap). Reported 
values represent the means and standard deviations of three independent experiments $(n=3)$. Indicated differences show the significant differences using an unpaired Student's t test. Two $\left(^{* *}\right)$ and four $\left(^{* * *}\right)$ asterisks denote a p-value lower than or equal to 0.01 and 0.0001 , respectively. (C) Same as (B), but rapamycin was added one hour before induction.

\section{Figure 4. Targeting dCas9 to specifically block PHO5 AS transcription enhances} induction kinetics of the PHO5 gene. (A) Chromatin immunoprecipitation (ChIP) analysis of dCas9 binding at the PHO5 gene locus. Immunoprecipitated DNA was quantified by qPCR with primers specific for different regions of the PHO5 promoter (Nucleosomes -4 and -1 ) and ORF regions. Both strains were transformed with a dCas9 expressing plasmid, while the CRISPRi strain was additionaly transformed with a plasmid expressing a gRNA targeted to strand-specifically block PHO5 AS transcription and the control strain with the corresponding empty plasmid. Nucl nucleosome, No Ab - no antibody ChIP control. (B) Levels of PHO5 AS transcribed at the PHO5 promoter and ORF regions in Nrd1-AA strain with or without addition of rapamycin (to deplete $\mathrm{Nrd1}$ and induce AS transcription) and an active CRISPRi system. RT-qPCR values were normalized to SCR1 mRNA. Reported values represent the means and standard deviations of three independent experiments $(n=$ 3). Indicated differences show the significant differences using an unpaired Student's $t$ test. Two $\left(^{* *}\right)$ and four $\left.{ }^{(* * *}\right)$ asterisks denote a p-value lower than or equal to 0.01 and 0.0001 , respectively. (C) Levels of PHO5 AS and mRNA transcripts in the CRISPRi and the corresponding control strain, monitored by RT-qPCR as in (B). Strains are Nrd1-AA with the absence of rapamycin (wild-type equivalent). (D) Levels of PHO5 mRNA in the CRISPRi and the corresponding control strain upon induction through phosphate starvation monitored by RT-qPCR as in (B). Strains are same as in (C). Reported values represent the means and standard deviations of three independent experiments $(n=3)$. Indicated differences show the significant differences using an unpaired Student's t test. One $\left({ }^{*}\right)$ and two $\left(^{* *}\right)$ asterisks denote a $p$-value lower than or equal to 0.05 and 0.01 , respectively. (E) Same as (D), but acid phosphatase induction kinetics were monitored by measuring acid phosphatase activity with whole cells. 
Figure 5. PHO5 AS elongation negatively affects kinetics of histone removal at the PHO5 gene promoter upon induction. (A) ChIP analysis of histone H3 binding at nucleosome -2 of the PHO5 gene promoter in wild-type BMA41 (wt) and corresponding rrp6s and air1 $\Delta$ air2 $\Delta$ cells upon induction through phosphate starvation. Immunoprecipitated DNA was quantified by qPCR and normalized to a control genomic region adjacent to the PHO5 gene locus. (B) Scheme of the PHO5 gene promoter region. Nucleosomes are denoted by circles, Pho4 binding sites by squares (gray - low affinity, black - high affinity) and the TATA box by the letter T. Site of cleavage with the Clal restriction enzyme is denoted by a black arrow. (C) Kinetics of PHO5 promoter opening monitored by Clal accessibility at nucleosome -2 after induction as in $(A)$.

Figure 6. PHO5 AS elongation affects PHO5 gene induction via histone acetylation. (A) Heatmap of the PHO5 gene locus in wild-type W303 (wt), rpd34, rrp6 $\Delta$, and rrp6 $\Delta r p d 3 \Delta$ mutant cells. Snapshot of tilling arrays intensities from (14) at the PHO5 locus for the Watson (W, upper half) and the Crick (C, lower half) strands. Three replicates of each strain are represented. A darker signal depicts a higher score of RNA expression. The red vertical lines represent the inferred coding and non-coding genes boundaries. (B) Acid phosphatase induction kinetics in wild-type W303 (wt) and the corresponding rrp6 $\Delta$, rpd3 $\Delta$ and rrp64rpd3 $\Delta$ cells upon induction through phosphate starvation. Reported values represent the means and standard deviations of three independent experiments $(n=3)$. (C) As in (B), but for wild-type BY4741 (wt) and the corresponding $\operatorname{rrp} 6 \Delta, \mathrm{gcn} 5 \Delta$ and $\operatorname{rrp} 6 \Delta g c n 5 \Delta$ mutant cells.

Figure 7. Chromatin remodeling at the $\mathrm{PHO5}$ gene promoter is negatively affected by PHO5 AS elongation. (A) Snapshot of the PHO5 gene locus in Nrd1-AA and the corresponding rpd3 3 strain from MNase-seq, ATAC-seq and Sth1-ChEC experiments. Data is from (12). (B) Proposed model for how AS RNA regulates transcription of the $\mathrm{PHO5}$ gene via remodeling of promoter chromatin structure. In wild-type cells, antisense RNA transcription is terminated by the NNS complex and degraded by the RNA exosome. Histones at the PHO5 gene promoter are acetylated by Gcn5 and serve as docking sites for recruitment of the chromatin remodeling complex RSC, thus enabling physiological kinetics of promoter opening and gene 
induction. In rrp6 4 cells, read-through of the AS transcript into the PHO5 promoter region results in increased recruitment of the histone deacetylase $\mathrm{Rpd} 3$ and subsequently in hypoacetylation and decreased recruitment of RSC. This results in delayed kinetics of promoter opening and induction of the PHO5 gene. (C) Acid phosphatase induction kinetics in Sth1-AA and the corresponding rrp6 $\Delta$ cells upon induction through phosphate starvation with (+Rap) or without addition of rapamycin (-Rap). Reported values represent the means and standard deviations of three independent experiments $(n=3)$.

\section{Figure S1. Non-coding transcripts CUT025 and SUT446 are transcribed at the}

PHO5 gene locus. (A) A heatmap summarising tiling array expression data at the PHO5 gene locus in wild type W101 (wt) and corresponding rrp6 $\Delta$ cells. Data is from (34) and is visualized with the SGV Genomics Viewer (71). (B) Levels of SUT446 in wild type BMA41 (wt) and corresponding rrp6 4 mutant cells upon induction through phosphate starvation. RT-qPCR values were normalized to PMA1 mRNA and expressed relative to transcript abundance in wild-type cells at repressive conditions ( $0 \mathrm{~h}$ of induction), which was set to 1 .

Figure S2. Delayed induction kinetics of the PHO5, but not the lacZ gene under regulation of the PHO5 promoter in rrp64 mutant cells. (A) Acid phosphatase induction kinetics in wild-type BMA41 (wt) and corresponding mutant cells upon induction through phosphate starvation. The strain rrp6Y361A carries a point mutation at the RRP6 genomic locus which abolishes exonuclease activity of Rrp6. Plasmid pCEN-RRP6 is a centromeric plasmid which carries the RRP6 gene under regulation of its native promoter. Reported values represent the means and standard deviations of three independent experiments $(n=3)$. (B) Same as $(A)$, but for wild type and corresponding rrp6 4 mutant cells from the BY4741 genetic background. (C) Same as (A), but for wild type and corresponding rrp64 mutant cells from the LPY917 genetic background. (D) Beta-galactosidase induction kinetics in wild-type BMA41 (wt) and corresponding rrp6 $\Delta$ cells transformed with a reporter plasmid pP5Z carrying the lacZ gene under the control of the PHO5 promoter upon induction through phosphate starvation. Reported values represent the means and standard deviations of three independent experiments $(n=3)$. 
A
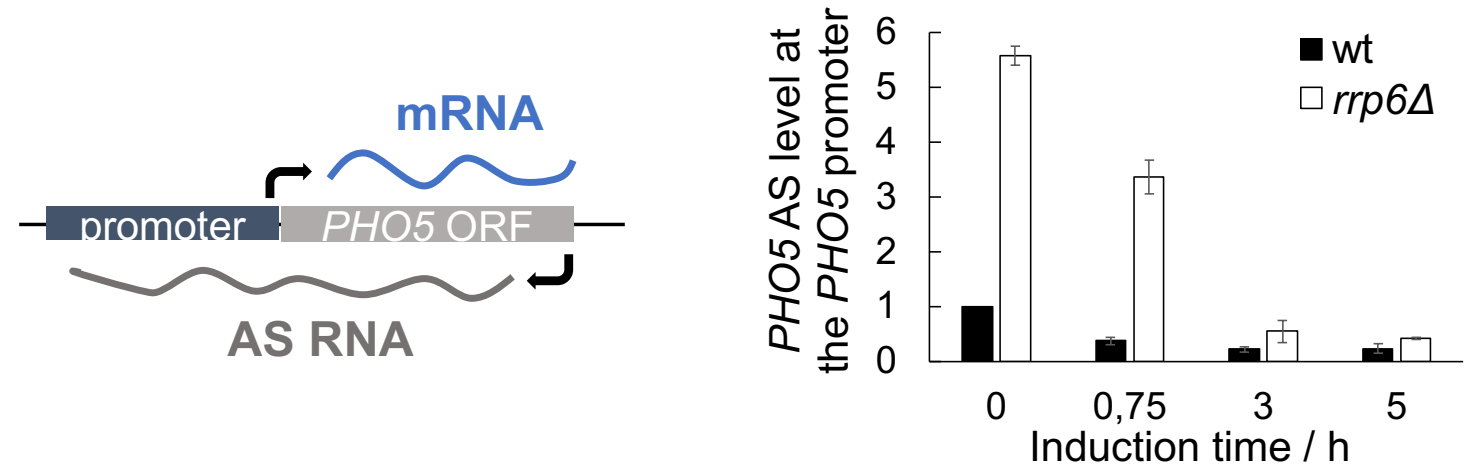

B

RNA-seq

RNAPII PAR-CLIP
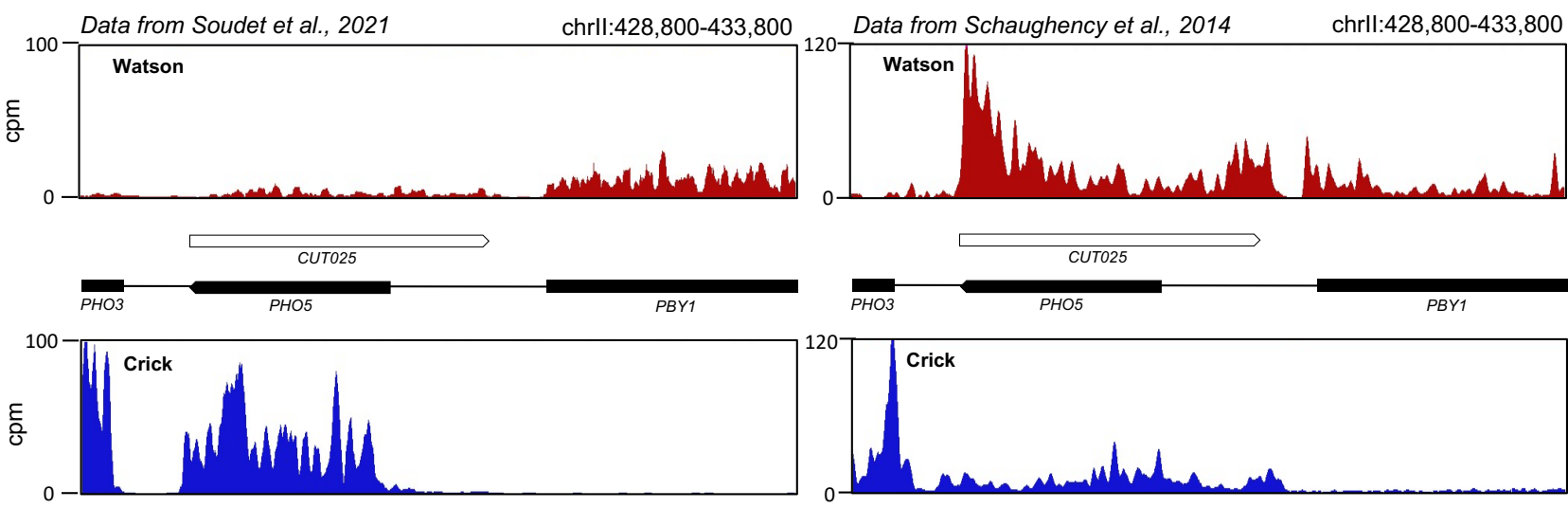

C
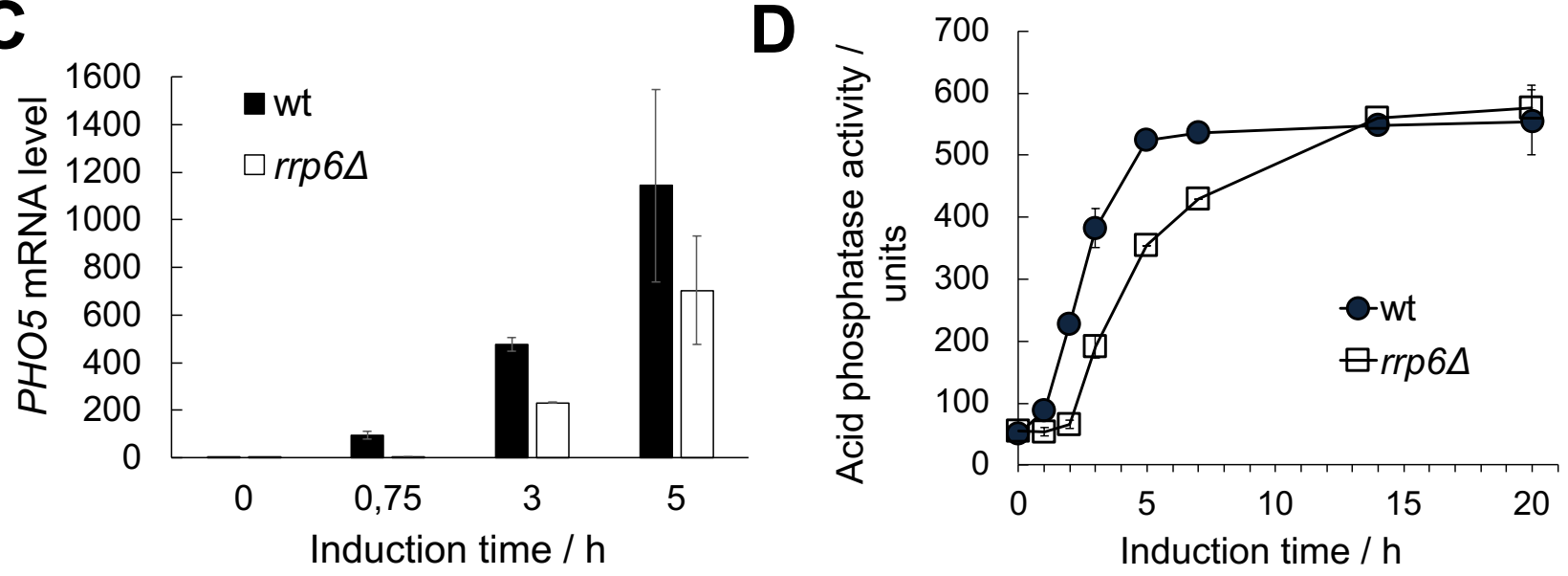

Figure 1 
A
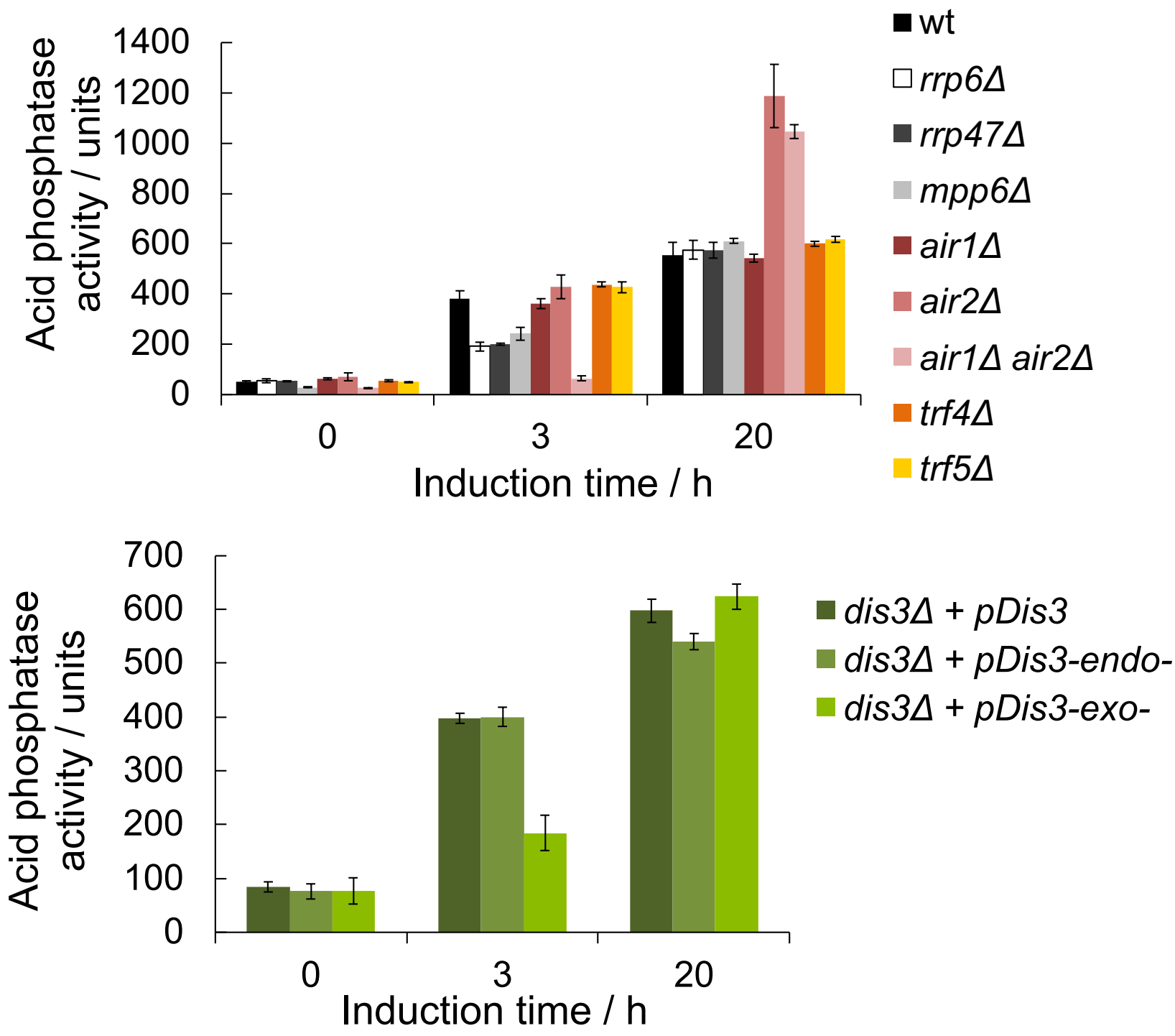
A

-Rap

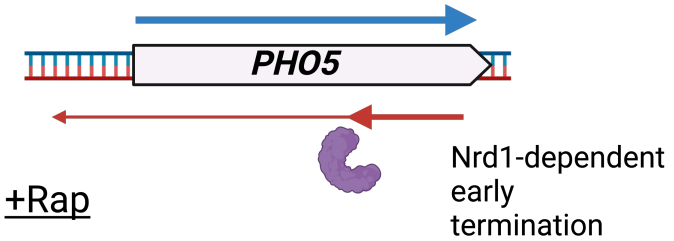

Time in Rap (min) $\quad 0^{\prime} \quad 60^{\prime} \quad 120^{\prime} \quad 0^{\prime} \quad 60^{\prime} \quad 120^{\prime}$

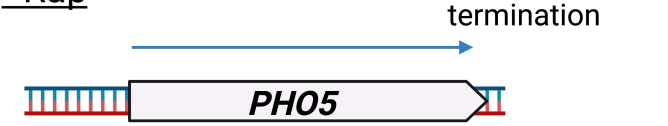

Anchor

away of

Nrd1

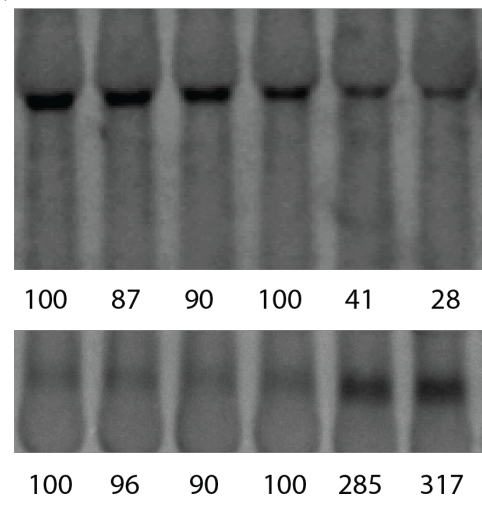

PHO5 S

PHO5 AS
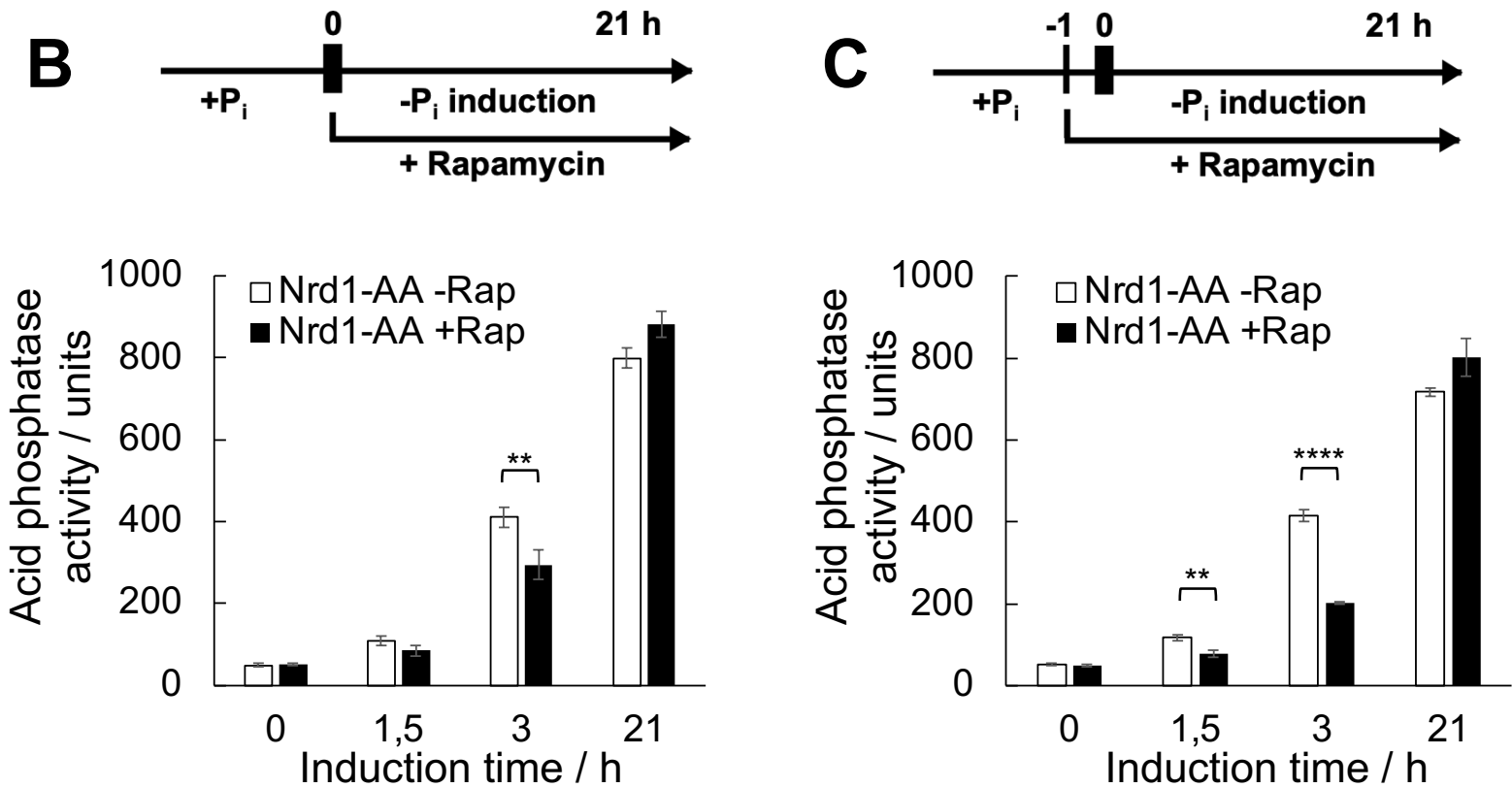

Figure 3 

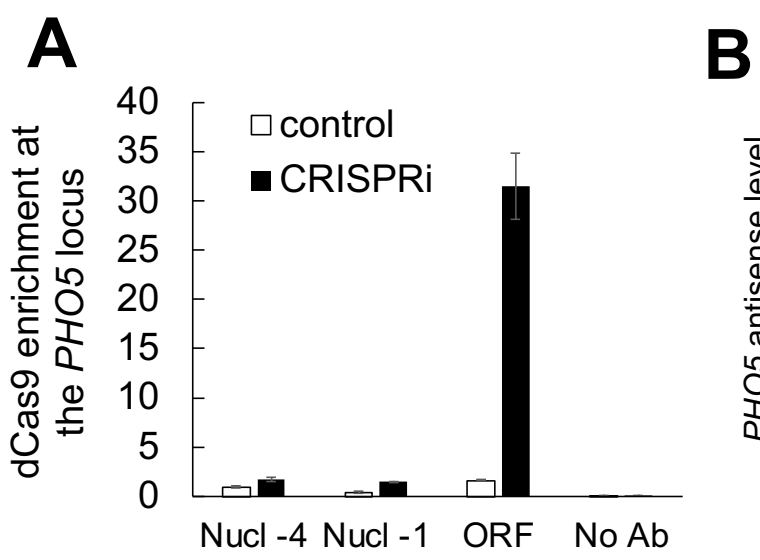

$\square$ control

- CRISPRi

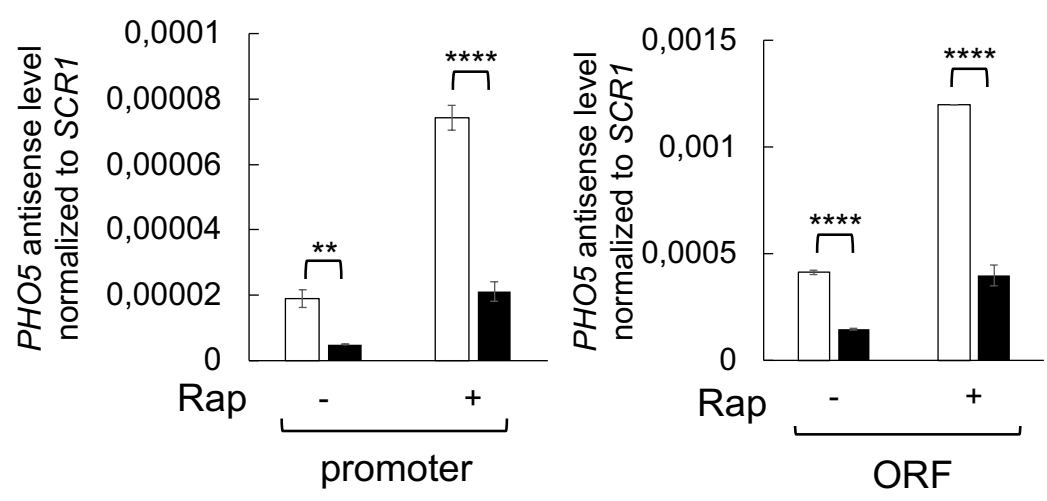

C
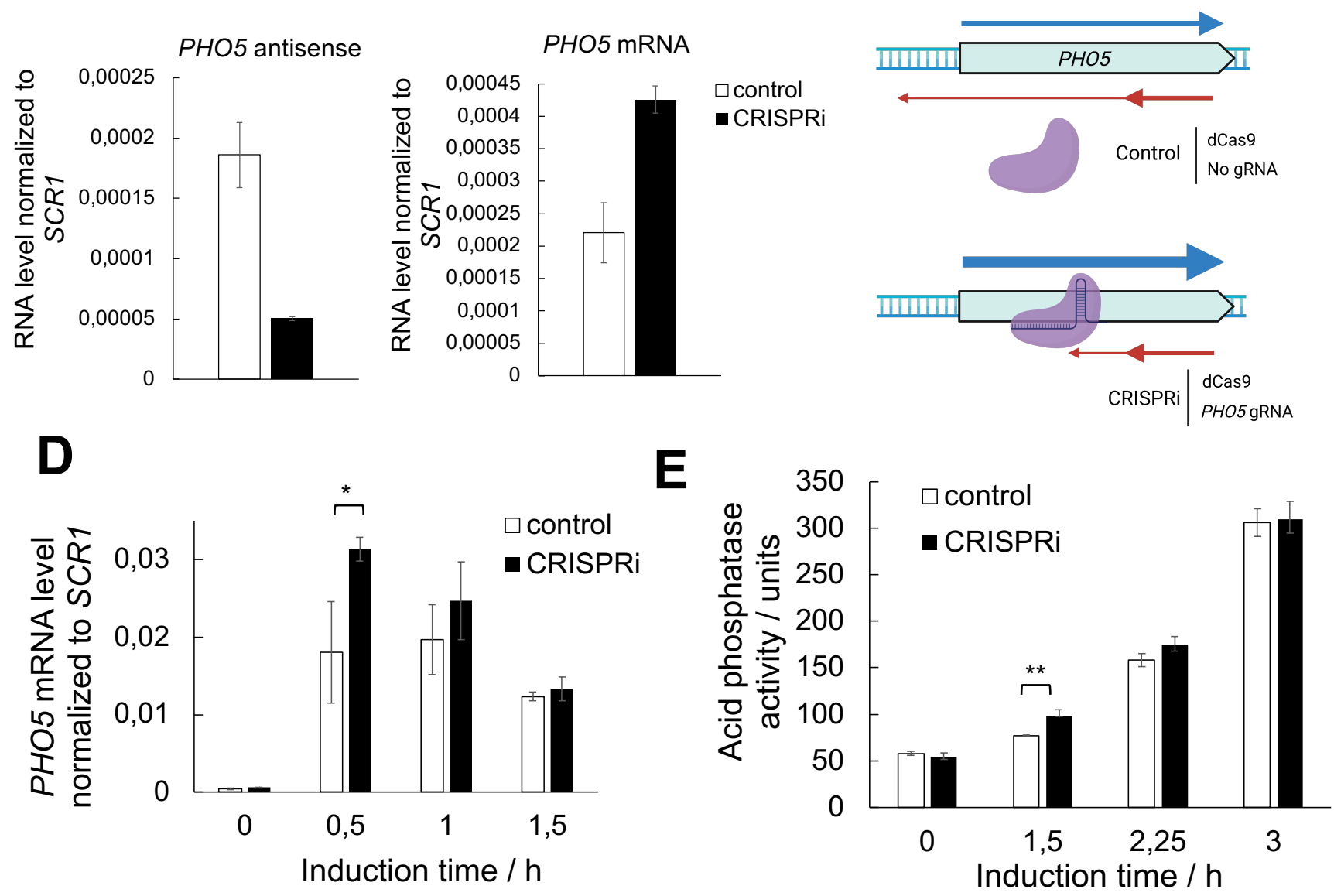

Figure 4 
A

PHO5 UASp2 (Nucl -2)

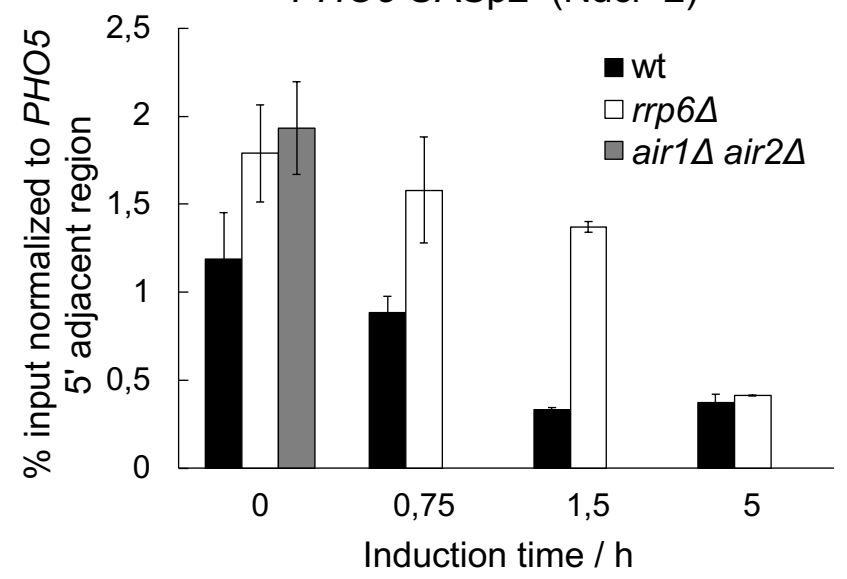

B

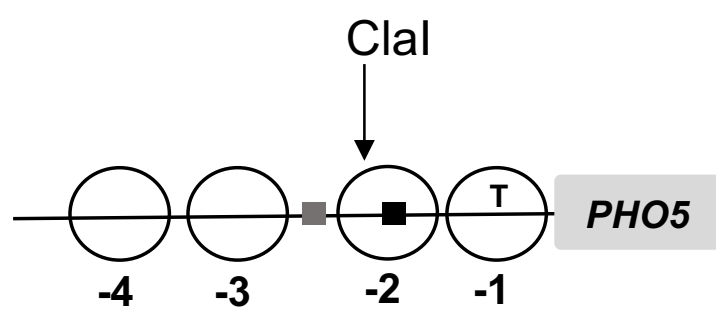

C

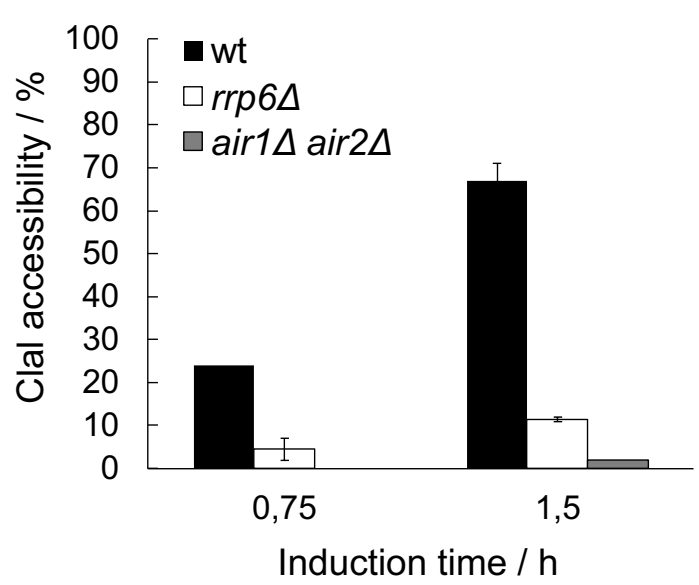

$\frac{1,5 \mathrm{~h} \text { induction }}{\text { wt } \quad \operatorname{rrp} 6 \Delta \text { air1 } \Delta \text { air2 } \Delta}$

Closed chromatin

Open chromatin

$63 \quad 11 \quad 2 \quad$ Clal accessibility (\%) 
A

B
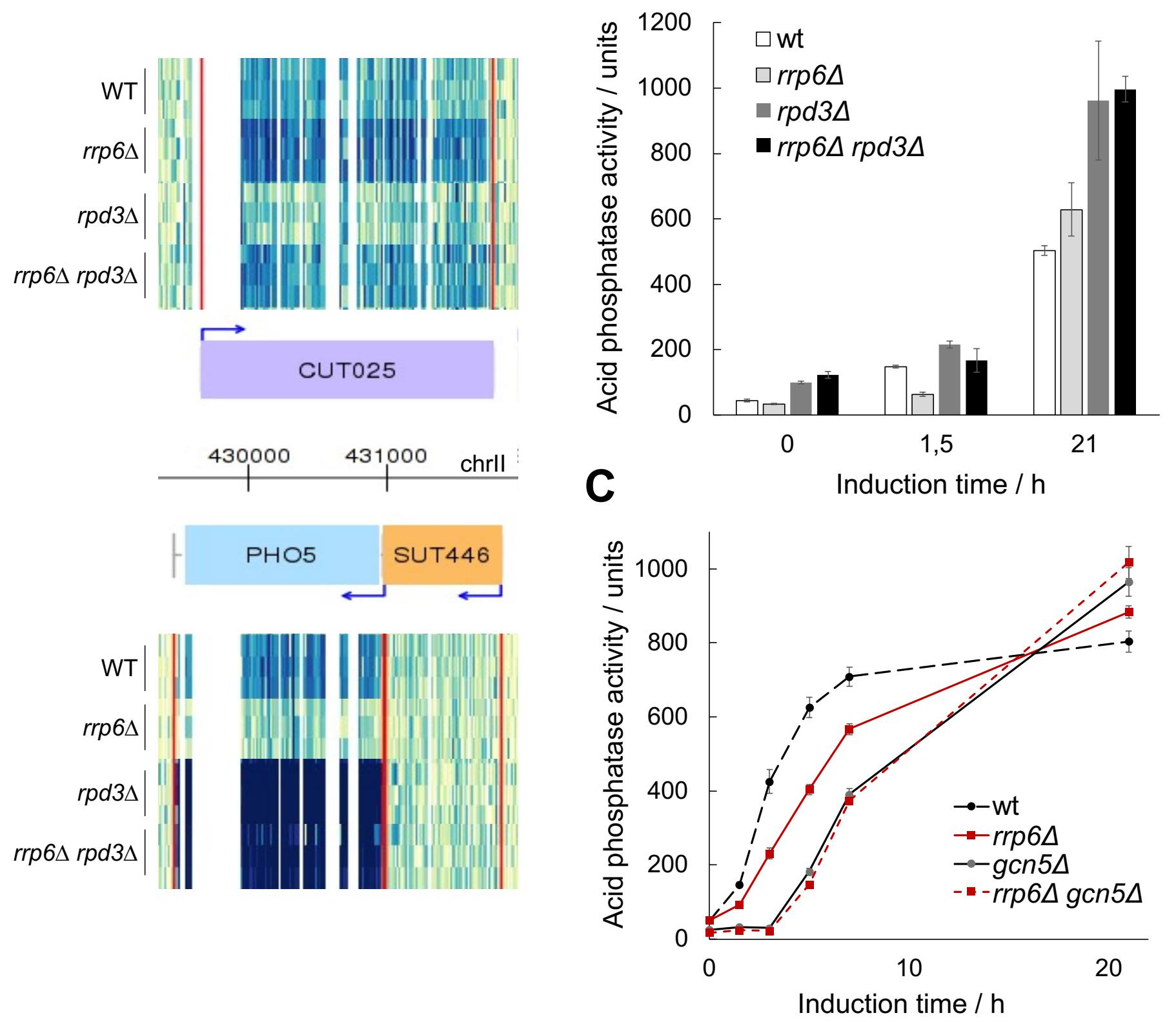

Figure 6 
A

Nrd1-AA

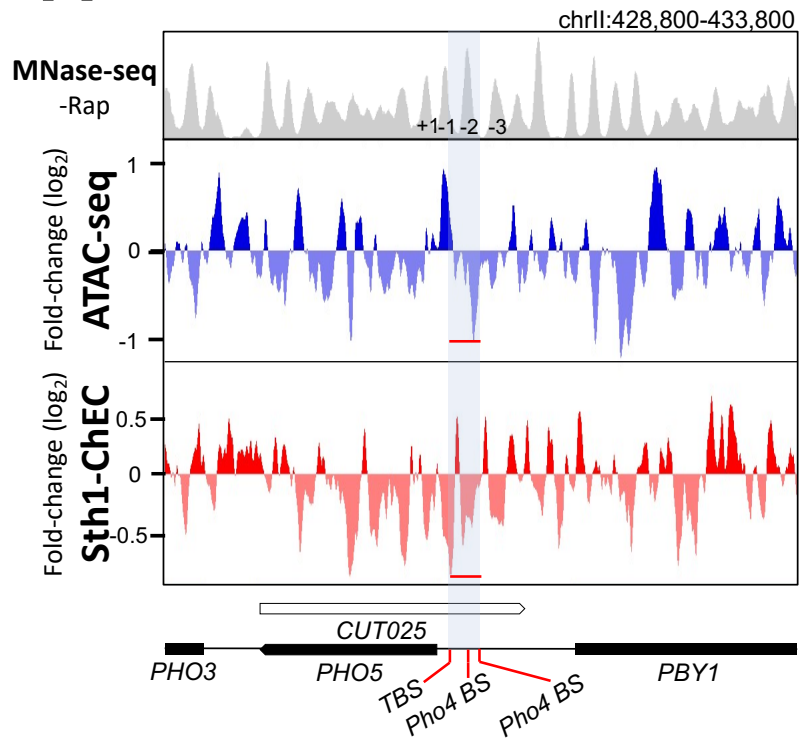

B

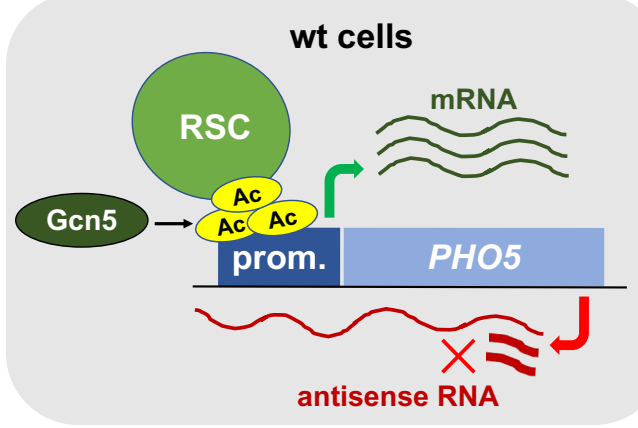

Nrd1-AA rpd3 $\Delta$

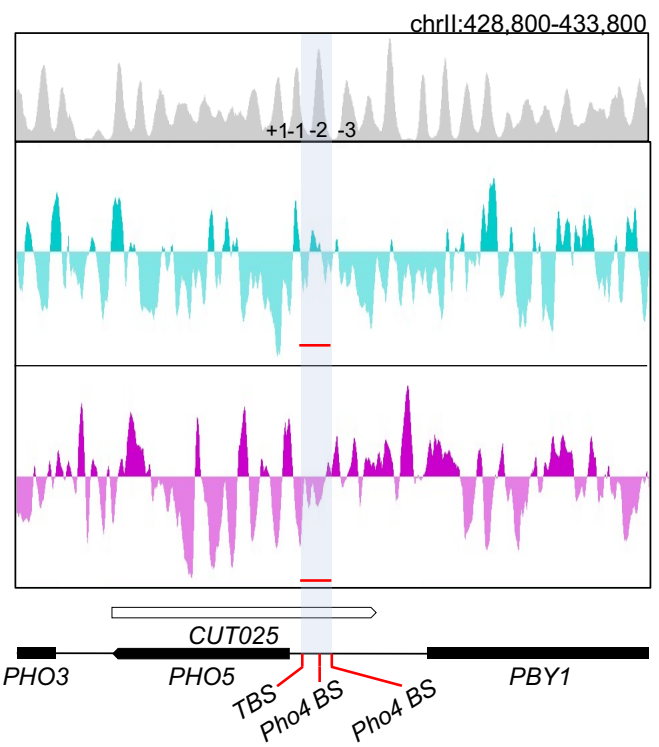

rrp6 $\Delta$ cells

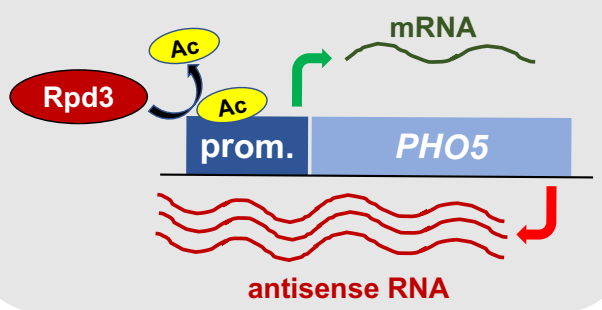

C

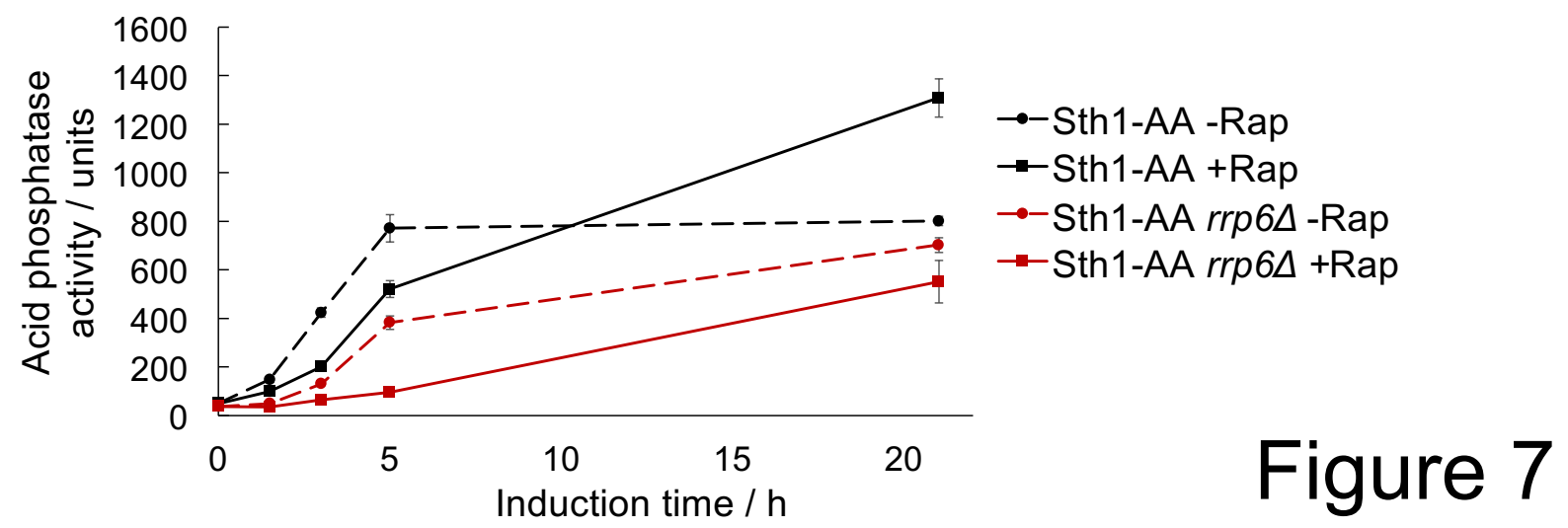

